\title{
On the consistency between nearest-neighbor peridynamic discretizations and discretized classical elasticity models
}

\author{
Pablo Seleson ${ }^{\mathrm{a}, *}$, Qiang Du ${ }^{\mathrm{b}}$, Michael L. Parks ${ }^{\mathrm{c}}$ \\ ${ }^{a}$ Computer Science and Mathematics Division, Oak Ridge National Laboratory, One Bethel Valley \\ Road, P.O. Box 2008, MS-6211, Oak Ridge, TN 37831-6211. \\ ${ }^{b}$ Department of Applied Physics and Applied Mathematics, Columbia University, New York, NY \\ $1002 \%$. \\ ${ }^{c}$ Center for Computing Research, Sandia National Laboratories, P.O. Box 5800, MS-1320, \\ Albuquerque, NM 87185-1320.
}

\begin{abstract}
The peridynamic theory of solid mechanics is a nonlocal reformulation of the classical continuum mechanics theory. At the continuum level, it has been demonstrated that classical (local) elasticity is a special case of peridynamics. Such a connection between these theories has not been extensively explored at the discrete level. This paper investigates the consistency between nearest-neighbor discretizations of linear elastic peridynamic models and finite difference discretizations of the Navier-Cauchy equation of classical elasticity. While nearest-neighbor discretizations in peridynamics have been numerically observed to present grid-dependent crack paths or spurious microcracks, this paper focuses on a different, analytical aspect of such discretizations. We demonstrate that, even in the absence of cracks, such discretizations may be problematic unless a proper selection of weights is used. Specifically, we demonstrate that using the standard meshfree approach in peridynamics, nearest-neighbor discretizations do not reduce, in general, to discretizations of corresponding classical models. We study nodal-based quadratures for the discretization of peridynamic models, and we derive quadrature weights that result in consistency between nearest-neighbor discretizations of peridynamic models and discretized classical models. The quadrature weights that lead to such consistency are, however, model-/discretization-dependent. We motivate the choice of those quadrature weights through a quadratic approximation of displacement fields. The stability of nearest-neighbor peridynamic schemes is demonstrated through a Fourier mode analysis. Finally, an approach based on a normalization of peridynamic constitutive constants at the discrete level is explored. This approach results in the desired consistency for onedimensional models, but does not work in higher dimensions. The results of the work presented in this paper suggest that even though nearest-neighbor discretizations should be avoided in peridynamic simulations involving cracks, such discretizations are viable, for example for verification or validation purposes, in problems characterized by smooth deformations. Moreover, we demonstrate that better quadrature rules in peridynamics can be obtained based on the functional form of solutions.
\end{abstract}

Keywords: peridynamics, meshfree method, consistency, nodal-based quadratures, classical finite differences, Navier-Cauchy equation of classical elasticity 2010 MSC: 45K05, 65R20, 70C20, 74B05, 74R99, 74S20 


\section{Introduction}

Nonlocal continuum theories have been proposed in past years as generalizations of classical (local) theories based on partial differential equations (PDEs). Two fields in which nonlocal models have drawn particular attention are continuum mechanics and

5 transport. In the former, nonlocal models allow representation of microscopic detail through the introduction of length scales not present in classical PDEs 1, 2, 3, as well as modeling material discontinuities, such as cracks, by utilizing integral operators that lack differentiability assumptions on displacement fields that are required for classical PDEs 4, 5. In the latter, nonlocal models enable the description of anomalous mass

10 diffusion and transport, as well as anomalous heat transfer, which is not well characterized by classical models $[6,7,8,9,10$.

The peridynamic (PD) theory of solid mechanics is a nonlocal reformulation of the classical continuum mechanics theory, based on integro-differential equations. Constitutive PD models do not require the spatial differentiability assumption on displacement

15 fields inherent to classical constitutive relations, and thus are directly applicable to the simulation of material failure and damage. Examples of this include: failure and damage in composites [11, 12, 13, 14, 15, crack propagation and branching [16, 4, 17, crack nucleation [5, 18, impact damage [19, 20, 21, 22, polycrystal fracture [23, 24, structural health monitoring [25, and damage in concrete [26, among others.

20 A fundamental characteristic of elastic PD models is that they reduce to classical elastic models in the limit of vanishing nonlocality, for either smooth deformations [27, 28] or even for non-smooth solutions of nonlocal models [29], or when higher-order gradients of the deformation, whereas defined, are negligible [2, 30. Common PD simulations of engineering systems employ the meshfree approach proposed in 20, which 25 directly approximates strong forms of governing equations. However, the relation between discretizations of strong forms of PD equations and discretizatized classical PDEs remains underexplored. Recently, Tian and Du presented studies regarding consistency between nonlocal and local models, as follows. In [31, one-dimensional finite element and quadrature-based finite difference discretizations of nonlocal models were investigated,

30 showing that the limiting behavior of the continuum model may or may not be preserved for various discrete approximations. Then, in 32 , they demonstrated that Galerkin finite element approximations of nonlocal models are asymptotically compatible with classical local models, as long as the finite element space contains continuous piecewise linear functions or as long as the mesh size decreases faster than the PD horizon for piecewise 35 constant finite elements. The results in 32 are valid for general linear elastic PD models (both linearized bond-based and state-based models) in multidimensional space and for finite element methods based on general, possibly nonuniform, meshes. In [33, the notion of asymptotically compatible schemes has also been illustrated for quadrature-based collocation type discretizations. Connections between the meshfree discretization of PD

\footnotetext{
*Corresponding author

Email addresses: selesonpd@ornl.gov (Pablo Seleson), qd2125@columbia.edu (Qiang Du), mlparks@sandia.gov (Michael L. Parks)

Preprint submitted to Computer Methods in Applied Mechanics and Engineering

July 12, 2016
} 
40 models with approximations of classical continuum models based on the reproducing kernel particle method (RKPM) have also been discussed in [34. Related numerical studies, referred to as $\delta$-convergence, have been presented in 35 .

In this paper, we concern ourselves with discretizations of strong forms of PD equilibrium equations. Specifically, we present nodal-based quadratures for discretization

45 of PD governing equations (of which meshfree discretizations are a special case), and we investigate relations between nearest-neighbor discretizations of PD models and finite difference discretizations of classical equations in one and two dimensions, which extend some of the results of [31]. We show that, under the current practice for meshfree methods in PD simulations, nearest-neighbor discretizations of PD models are, in

50 general, inconsistent with corresponding classical discrete equations. We demonstrate, however, that nearest-neighbor discretizations using generalized nodal-based quadratures can recover discretizations of classical models, for the right choice of quadrature weights. Those quadrature weights are model- and discretization-dependent, although their choice can be motivated through a quadratic approximation of displacement fields. Modifying

55 quadrature weights of PD discrete equations has also been done in [36], in the context of surface-effect corrections for PD models. The stability of the nearest-neighbor discrete schemes studied here is also established. Additional approaches that employ normalization of PD constitutive constants at the discrete level are investigated. These approaches seem effective for one-dimensional models, but do not work in higher dimensions.

60 The organization of this paper is as follows. In Section 2, we provide a brief overview of the PD theory and introduce a nodal-based quadrature discretization, which generalizes a common meshfree descretization of PD models. In Section 3 we study onedimensional linear elastic bond-based PD models and propose two methods to achieve consistency: (1) selection of model-/discretization-dependent quadrature weights and

65 (2) use of normalizations of constitutive constants at the discrete level. We demonstrate in Section 3.2. that the choice of quadrature weights for the first method can be motivated by a quadratic approximation of displacement fields. In Section 4 , we extend the analysis to two-dimensional linear elastic state-based PD models, demonstrating that from the two methods proposed in Section 3 , the first one is also effective in two-

70 dimensions, whereas the second one is not. We derive in Section 4.2 the stability of the studied two-dimensional nearest-neighbor scheme, based on a Fourier mode analysis. As for the one-dimensional models, in Section 4.3 we motivate the choice of quadrature weights through a quadratic approximation of displacement fields. We demonstrate in Section 4.4 that, in contrast to the one-dimensional case, a normalization of PD consti-

75 tutive constants at the discrete level does not provide in higher dimensions an effective method to achieve the consistency studied in this paper. Concluding remarks are given in Section 5

\section{The peridynamic theory}

The peridynamic theory [1, 37, 38, 39] has been proposed as a nonlocal generalization so of classical continuum mechanics, suitable for material failure and damage simulation.

Given a bounded body $\mathcal{B} \subset \mathbb{R}^{d}$ with $d=1,2$, or 3 the dimension, the state-based PD equilibrium equation for $\mathbf{x} \in \mathcal{B}$ is

$$
\int_{\mathcal{B}}\left\{\underline{\mathbf{T}}[\mathbf{x}]\left\langle\mathbf{x}^{\prime}-\mathbf{x}\right\rangle-\underline{\mathbf{T}}\left[\mathbf{x}^{\prime}\right]\left\langle\mathbf{x}-\mathbf{x}^{\prime}\right\rangle\right\} d \mathbf{x}^{\prime}=-\mathbf{b}(\mathbf{x}),
$$


where $\mathbf{b}$ is a prescribed body force density field and $\underline{\mathbf{T}}$ is the force state field. The concept of a PD state has been introduced in [37] as a scalar, vector, or tensor operator that operates on bonds, which are relative position vectors in the reference configuration. The notation $\boldsymbol{\xi}=\mathbf{x}^{\prime}-\mathbf{x}$ is commonly used to represent the bond connecting the material point $\mathbf{x}$ to the material point $\mathbf{x}^{\prime}$. A force state is a vector-valued operator that maps bonds to force per unit volume squared. The spatial dependence of the force state field is indicated in square brackets and the bond this field operates on is included within angle brackets. It is assumed that a material point $\mathbf{x}$ directly interacts with its neighborhood,

$$
\mathcal{H}(\mathbf{x}, \delta):=\left\{\mathbf{x}^{\prime} \in \mathbb{R}^{d}:\left\|\mathbf{x}^{\prime}-\mathbf{x}\right\| \leqslant \delta\right\},
$$

which represents a closed line segment, disk, or ball in 1D, 2D, or 3D, respectively. Consequently, $\underline{\mathbf{T}}[\mathbf{x}]\langle\boldsymbol{\xi}\rangle=\mathbf{0} \forall \boldsymbol{\xi} \in \mathbb{R}^{d}:\|\boldsymbol{\xi}\|>\delta$. The parameter $\delta$ in (2) is referred to as the $P D$ horizon and represents a length scale.

The PD length scale can be used to connect the PD and classical theories. Specifically, it can be shown for an elastic PD material that if: (i) the deformation is twice continuously differentiable in space and if (ii) the force state is a continuously differentiable function of the deformation and the position, Equation (11) reduces to the PDE

$$
\nabla \cdot \nu^{0}(\mathbf{x})=-\mathbf{b}(\mathbf{x})
$$

in the limit as $\delta \rightarrow 0$ [27. In (3), $\boldsymbol{\nu}^{0}$ is the collapsed PD stress tensor, and it can be 85 shown that it represents a Piola-Kirchhoff stress tensor in the classical theory. Equation (3) thus represents a classical Cauchy's equation of equilibrium, which demonstrates the convergence of the PD theory to the classical PDE-based theory in the local limit. Moreover, in works such as [29, the convergence of (nonlocal) solutions of PD problems to corresponding (local) solutions of PDE-based problems has been demonstrated without requiring the nonlocal solutions to be smooth.

\subsection{A nodal-based quadrature discretization of peridynamic models}

Let a body $\mathcal{B} \subset \mathbb{R}^{d}$ be discretized with a set of $N$ nodes $\left\{\mathbf{x}_{i}\right\}_{i=1, \ldots, N}$. Using a tessellation of the domain, we define a volume region (or cell) $\tau_{j}$ around each node $j$ with corresponding volume $V_{j}$. A nodal-based quadrature discretization of (1) at $\mathbf{x}_{i}$ is given by

$$
\sum_{j \in \mathcal{F}_{i}}\left\{\underline{\mathbf{T}}\left[\mathbf{x}_{i}\right]\left\langle\mathbf{x}_{j}-\mathbf{x}_{i}\right\rangle-\underline{\mathbf{T}}\left[\mathbf{x}_{j}\right]\left\langle\mathbf{x}_{i}-\mathbf{x}_{j}\right\rangle\right\} V_{j}^{(i)}=-\mathbf{b}_{i},
$$

where $V_{j}^{(i)}$ is the quadrature weight associated with node $j$ in the equation of node $i$, and $\mathbf{b}_{i}:=\mathbf{b}\left(\mathbf{x}_{i}\right)$. We define $\mathcal{F}_{i}$ as the family of $i$, which contains the nodes that directly interact with node $i$. For the purpose of this paper, $\mathcal{F}_{i}$ contains the set of quadrature 95 nodes in the equation of node $i$.

Special cases from the literature of the discretization scheme (4) are as follows:

(a) Meshfree method: This method was proposed in 20. It is obtained by taking $\mathcal{F}_{i}$ as the set of all nodes $j \neq i$ such that $\mathbf{x}_{j} \in \mathcal{H}\left(\mathbf{x}_{i}, \delta\right)$ and the quadrature weights as $V_{j}^{(i)}=V_{j}$. 
(b) Partial-volume method: This method has been presented in [40, 41, 42, 30, as an improvement of the meshfree method. Quadrature weights in this method are chosen as volumes (in 3D), areas (in 2D), or lengths (in 1D) of intersections (or as their approximations) between neighbor cells and the neighborhood of a given node. The family of a node in this method often includes neighboring nodes outside the neighborhood of that node having a cell that overlaps that neighborhood.

(c) Quadrature-based finite difference method: This method was proposed in 31 for one-dimensional linear PD models. It employs piecewise polynomial basis functions, satisfying partitions of unity, in combination with finite difference approximations to decompose PD spatial integrals into weighted summations of nodal displacement differences.

\section{One-dimensional bond-based peridynamic models}

Let a one-dimensional linear elastic bond-based static PD model be given by the force state

$$
\underline{\mathrm{T}}[x]\langle\xi\rangle=\frac{1}{2} \frac{c}{|\xi|^{\alpha}}(u(x+\xi)-u(x)), \quad \forall x \in \mathcal{B}, \forall \xi \in \mathcal{H}(0, \delta),
$$

where $u$ is the displacement field, $c$ is a constitutive constant, and $\alpha<3$ is a parameter. The derivations in this section are presented for material points $x \in \mathcal{B}$ in the bulk of the body, i.e., farther than $\delta$ from the boundary $\partial \mathcal{B}$. For such points, $\mathcal{H}(x, \delta)=[x-\delta, x+\delta] \subset \mathcal{B}$ and the $\mathrm{PD}$ equilibrium equation (1) can be written as

$$
\int_{x-\delta}^{x+\delta} \frac{c}{\left|x^{\prime}-x\right|^{\alpha}}\left(u\left(x^{\prime}\right)-u(x)\right) d x^{\prime}=-b(x) .
$$

An expression for $c$ can be obtained from the consistency with the local limit given by the classical local equation

$$
K \frac{d^{2} u}{d x^{2}}(x)=-b(x)
$$

Indeed, we have the following relation satisfied,

$$
c=\frac{(3-\alpha) K}{\delta^{3-\alpha}} .
$$

\subsection{A nearest-neighbor discretization of peridynamic models}

Let us assume a discretization of a one-dimensional domain using a uniform grid with grid spacing $h$. Following the discretization method described in Section 2.1, the discretization of 6 at node $x_{i}$ is given by

$$
\sum_{j \in \mathcal{F}_{i}} \frac{(3-\alpha) K}{\delta^{3-\alpha}} \frac{1}{\left|x_{j}-x_{i}\right|^{\alpha}}\left(u_{j}-u_{i}\right) V_{j}^{(i)}=-b_{i},
$$

where $u_{i}:=u\left(x_{i}\right)$. A nearest-neighbor discretization of the PD model can then be written as

$$
\frac{(3-\alpha) K}{\delta^{3-\alpha}} \frac{u_{i+1}-2 u_{i}+u_{i-1}}{h_{5}^{\alpha}} V_{i \pm 1}^{(i)}=-b_{i} .
$$


Choosing the quadrature weight in 10 as

$$
V_{i \pm 1}^{(i)}=\left(\frac{\delta}{h}\right)^{3-\alpha} \frac{h}{3-\alpha},
$$

we recover a classical finite difference discretization of 77 :

$$
K \frac{u_{i+1}-2 u_{i}+u_{i-1}}{h^{2}}=-b_{i} .
$$

We now establish connections between (10) and the discretization methods from the literature discussed in Section 2.1

- Meshfree method: In the meshfree method [20, we choose (in 1D) $V_{i \pm 1}^{(i)}=h$. Then, a special choice of grid spacing is required to recover the classical discrete equation $(12)$, and it is given by

$$
h=\frac{\delta}{\sqrt[3-\alpha]{3-\alpha}} .
$$

This results in

$$
h=\left\{\begin{array}{cc}
\delta / \sqrt[3]{3} & \alpha=0 \\
\delta / \sqrt{2} & \alpha=1, \\
\delta & \alpha=2 .
\end{array}\right.
$$

We observe that the choice of $h=\delta$ for nearest-neighbor PD simulations does not in general result in the reduction of discrete PD equations to corresponding classical discrete equations. An exception, in this case, is when $\alpha=2$. A similar result has been observed for related nonlocal diffusion-type models in 43 .

- Partial-volume method: Let a discretization be given by $h=\delta / \gamma$ with $\gamma \in\left(\frac{1}{2}, \frac{3}{2}\right]$. In this case, the quadrature weight of the partial-volume method is chosen as the length of the intersection between the $i \pm 1$ cell and the neighborhood of node $i$, i.e., $V_{j}^{(i)}=\left(\gamma-\frac{1}{2}\right) h$ [41, 42, 30] (see Figure 11.

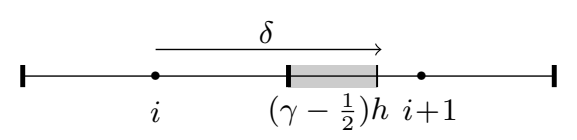

Figure 1: Illustration of the partial-volume method in a 1D nearest-neighbor discretization.

We can then write 10 as

$$
C(\gamma) K \frac{u_{i+1}-2 u_{i}+u_{i-1}}{h^{2}}=-b_{i}
$$

with

$$
C(\gamma):=\frac{(3-\alpha)\left(\gamma-\frac{1}{2}\right)}{\gamma^{3-\alpha}}
$$


To recover 12, we need $C(\gamma)=1$. This requires solving the equation

$$
\gamma^{3-\alpha}-(3-\alpha) \gamma+\frac{1}{2}(3-\alpha)=0
$$

for $\gamma$ in the range $\left(\frac{1}{2}, \frac{3}{2}\right]$, for a given value of $\alpha$. The resulting choices of grid spacing for $\alpha=0,1$, required to recover the local discrete equation, are:

$$
h=\left\{\begin{array}{cc}
\sim \delta / 0.56, \sim \delta / 1.38 & \alpha=0, \\
\delta & \alpha=1 .
\end{array}\right.
$$

In the case of $\alpha=2$, no value of grid spacing (for $\gamma$ in the range of interest) satisfies the required condition. In Figure 2, we plot $C(\gamma)$ in (14), for $\alpha=0,1,2$.

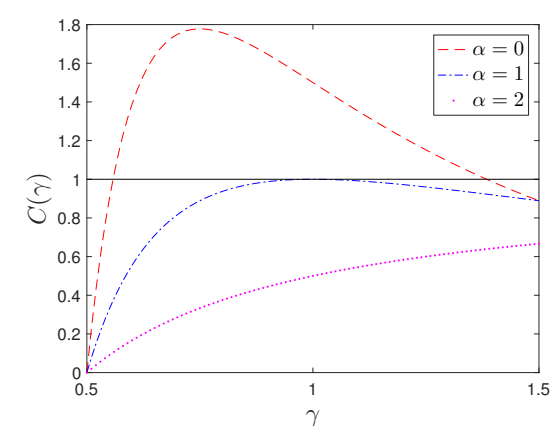

Figure 2: Profile of $C(\gamma)$ in 14 as a function of $\gamma$, for $\alpha=0,1,2$.

- Quadrature-based finite difference method: Let a discretization be given by $h>\delta$ and introduce a parameter $p$ representing a polynomial order of basis functions. In particular, let $p=0$ or $p=1$ for a choice of piecewise constant (PWC) or piecewise linear (PWL) basis functions, respectively. A nearest-neighbor quadrature-based finite difference discretization ( $c f$. [31]) of (6) can be obtained from (10) by choosing the quadrature weight

$$
V_{i \pm 1}^{(i)}=\left(\frac{\delta}{h}\right)^{\beta+p+1-\alpha} \frac{h}{\beta+p+1-\alpha},
$$

where the parameter $\beta \in[0,2]$ for PWC basis functions or $\beta \in[0,2)$ for PWL basis functions. In particular, we require $\beta>\alpha-(p+1)$. If $\beta=2-p$ (which is consistent with the constraint $\alpha<3$ ) the above quadrature weight reduces to the one in (11), and we recover the classical discrete equation $(12)$. Otherwise, a special choice of grid spacing is required to recover 12 , and it is given by

$$
h=\frac{\delta}{\left(\frac{\beta+p+1-\alpha}{3-\alpha}\right)^{\frac{1}{\beta+p-2}}} .
$$

We observe that the above expression for the grid spacing reduces to $h=\delta$ when $\beta=2-p$. 


\subsection{A quadrature based on a quadratic approximation of the displacement field}

Let a one-dimensional domain be discretized with a uniform grid of grid spacing $h=\delta$, having $N$ nodes: $\left\{x_{i}\right\}_{i=1, \ldots, N}$, and let the displacement field be approximated by a quadratic profile within the neighborhood of a given node $i$. Using a Lagrange interpolating polynomial, we express the displacement field as

$u\left(x^{\prime}\right)=\frac{\left(x^{\prime}-x_{i}\right)\left(x^{\prime}-x_{i+1}\right)}{2 h^{2}} u_{i-1}-\frac{\left(x^{\prime}-x_{i-1}\right)\left(x^{\prime}-x_{i+1}\right)}{h^{2}} u_{i}+\frac{\left(x^{\prime}-x_{i-1}\right)\left(x^{\prime}-x_{i}\right)}{2 h^{2}} u_{i+1}$,

for $x^{\prime} \in\left[x_{i}-\delta, x_{i}+\delta\right]$. Reordering terms, we can write 15 as

$$
u\left(x^{\prime}\right)=u_{i}+\frac{u_{i+1}-u_{i-1}}{2 h}\left(x^{\prime}-x_{i}\right)+\frac{1}{2} \frac{u_{i+1}-2 u_{i}+u_{i-1}}{h^{2}}\left(x^{\prime}-x_{i}\right)^{2} .
$$

A direct substitution into (6) (evaluated at $x_{i}$ ) gives the classical local discrete equation (12). A discretization based on a quadratic Lagrange interpolant of the displacement field using nodal values thus recovers, for $h=\delta$, the classical discrete equation.

To estimate the error in the approximation of the PD equation by the classical discrete one, we assume a displacement field with enough regularity. Employing a Taylor expansion, we can write the PD internal force density at $x_{i}$ as

$$
\int_{x_{i}-\delta}^{x_{i}+\delta} \frac{(3-\alpha) K}{\delta^{3-\alpha}} \frac{1}{\left|x^{\prime}-x_{i}\right|^{\alpha}}\left(u\left(x^{\prime}\right)-u_{i}\right) d x^{\prime}=K\left(\frac{d^{2} u}{d x^{2}}\left(x_{i}\right)+\frac{2}{4 !} \frac{3-\alpha}{5-\alpha} \frac{d^{4} u}{d x^{4}}\left(x_{i}\right) \delta^{2}+\ldots\right)
$$

and the corresponding discrete expression in classical elasticity as

$$
K \frac{u_{i+1}-2 u_{i}+u_{i-1}}{h^{2}}=K\left(\frac{d^{2} u}{d x^{2}}\left(x_{i}\right)+\frac{2}{4 !} \frac{d^{4} u}{d x^{4}}\left(x_{i}\right) h^{2}+\ldots\right) .
$$

We then have,

$$
\begin{aligned}
\int_{x_{i}-\delta}^{x_{i}+\delta} \frac{(3-\alpha) K}{\delta^{3-\alpha}} \frac{1}{\left|x^{\prime}-x_{i}\right|^{\alpha}}\left(u\left(x^{\prime}\right)-u_{i}\right) d x^{\prime}= & K \frac{u_{i+1}-2 u_{i}+u_{i-1}}{h^{2}} \\
& +\frac{2}{4 !} K\left(\frac{3-\alpha}{5-\alpha} \delta^{2}-h^{2}\right) \frac{d^{4} u}{d x^{4}}\left(x_{i}\right)+\ldots
\end{aligned}
$$

and, for $h=\delta$, the error estimate

$$
\begin{gathered}
\left|\int_{x_{i}-\delta}^{x_{i}+\delta} \frac{(3-\alpha) K}{\delta^{3-\alpha}} \frac{1}{\left|x^{\prime}-x_{i}\right|^{\alpha}}\left(u\left(x^{\prime}\right)-u_{i}\right) d x^{\prime}-K \frac{u_{i+1}-2 u_{i}+u_{i-1}}{h^{2}}\right| \leqslant \frac{K}{3 !(5-\alpha)}\left|\frac{d^{4} u}{d x^{4}}\left(x_{i}\right)\right| h^{2} \\
+\ldots=\mathcal{O}\left(h^{2}\right) .
\end{gathered}
$$

We now connect the above analysis with the quadrature weights in (11). Choosing $\delta=h$, we obtain from 11

$$
V_{i \pm 1}^{(i)}=\left\{\begin{array}{cc}
\frac{h}{3} & \alpha=0 \\
\frac{h}{2} & \alpha=1 \\
h & \alpha=2 \\
8 &
\end{array}\right.
$$


For the quadratic displacement field (16) and the choice of grid spacing $h=\delta$, we have

$$
\begin{aligned}
& \int_{x_{i}-h}^{x_{i}+h} \frac{(3-\alpha) K}{h^{3-\alpha}} \frac{1}{\left|x^{\prime}-x_{i}\right|^{\alpha}}\left(u\left(x^{\prime}\right)-u_{i}\right) d x^{\prime} \\
& =\frac{(3-\alpha) K}{h^{3-\alpha}}\left[\int_{x_{i}-h}^{x_{i}+h}\left|x^{\prime}-x_{i}\right|^{2-\alpha} d x^{\prime}\right] \frac{1}{2} \frac{u_{i+1}-2 u_{i}+u_{i-1}}{h^{2}},
\end{aligned}
$$

where the contribution of the second term on the right-hand side of (16) vanishes due to the symmetry of the integration range. Equation 18 can be written in the form of 100 with the quadrature weight given by

$$
V_{i \pm 1}^{(i)}=\frac{1}{2}\left[\int_{x_{i}-h}^{x_{i}+h}\left|x^{\prime}-x_{i}\right|^{2-\alpha} d x^{\prime}\right] \frac{1}{h^{2-\alpha}} .
$$

The integrand in 190 behaves as a constant for $\alpha=2$, as a piecewise linear function of $x^{\prime}$ for $\alpha=1$, and as a quadratic function of $x^{\prime}$ for $\alpha=0$. This suggests different quadrature rules for different values of $\alpha$, as follows. For $\alpha=0$, the Simpson's rule is considered, which assumes a quadratic approximation of integrands; for $\alpha=1$, the trapezoidal rule is considered, which assumes a linear approximation of integrands; and for $\alpha=2$, the rectangle method is considered, which assumes a constant approximation of integrands. Using those rules in (19) recovers (17). Furthermore, directly employing the Simpson's rule for $\alpha=0$, a composite trapezoidal rule for $\alpha=1$, or a composite rectangle method for $\alpha=2$ in (6) given $h=\delta$, we recover (12); the corresponding quadrature weights in those cases coincide with the ones in (17).

Remark 1. For $h=\delta$, the quadrature weights in 17) coincide with the quadrature weights of: (i) the meshfree method for $\alpha=2$; (ii) the partial-volume method for $\alpha=1$; and (iii) the quadrature-based finite difference method for all three values of $\alpha$, given $\beta=2-p$.

\subsection{An approach based on a discrete normalization of peridynamic constitutive constants}

Let the displacement field be quadratic. Using a Taylor expansion, we get from (6)

$$
\frac{1}{2}\left[\int_{x-\delta}^{x+\delta} \frac{c}{\left|x^{\prime}-x\right|^{\alpha}}\left(x^{\prime}-x\right)^{2} d x^{\prime}\right] \frac{d^{2} u}{d x^{2}}(x)=-b(x),
$$

where the term with a first derivative of the displacement field vanishes due to the symmetry of the integration range. Taking the PD constant as

$$
c=\frac{K}{\int_{0}^{\delta} \xi^{2-\alpha} d \xi}
$$

\footnotetext{
${ }^{1}$ In the composite trapezoidal rule for $\alpha=1$, we assume $u \in C^{2}$ so that the following limit holds $\lim _{x^{\prime} \rightarrow x_{i}} \frac{u\left(x^{\prime}\right)-u_{i}}{\left|x^{\prime}-x_{i}\right|}=0$.
} 
we obtain the classical local equation (7). Simultaneously discretizing the integral in 20 and the PD equilibrium equation at $x_{i}$ with the same nodal-based quadrature given in Section 2.1. we can write a nearest-neighbor discretization of (6) for $h=\delta$ as

$$
\frac{K}{h^{2-\alpha} V_{i \pm 1}^{(i)}} \frac{u_{i+1}-2 u_{i}+u_{i-1}}{h^{\alpha}} V_{i \pm 1}^{(i)}=-b_{i}
$$

which recovers the discrete local equation 12 . In this case, we have employed a normalization of the PD constant at the discrete level. A similar approach has been used in [44, 45. Another related approach has been discussed in [31, 33, where it was found for one-dimensional linear PD models that the local limits differ from the desired correct ones only by a multiplicative constant factor. Therefore, a scaling factor could possibly be employed in such models in the discrete schemes to correct the limit. The authors of those papers, however, warned that such a correction may not work for complex material models.

\section{Two-dimensional state-based peridynamic models}

Let a two-dimensional linear elastic state-based static PD model be given by a linearized linear peridynamic solid force state [37, 46,

$$
\underline{\mathbf{T}}[\mathbf{x}]\langle\boldsymbol{\xi}\rangle=\frac{c_{1}}{m} \underline{\omega}\langle\boldsymbol{\xi}\rangle \theta^{\operatorname{lin}}[\mathbf{x}] \boldsymbol{\xi}+\frac{c_{2}}{m} \underline{\omega}\langle\boldsymbol{\xi}\rangle \frac{\boldsymbol{\xi} \otimes \boldsymbol{\xi}}{\|\boldsymbol{\xi}\|^{2}}(\mathbf{u}(\mathbf{x}+\boldsymbol{\xi})-\mathbf{u}(\mathbf{x})),
$$

with $c_{1}$ and $c_{2}$ constants, and the linearized nonlocal dilatation given (in 2D) by

$$
\theta^{\operatorname{lin}}[\mathbf{x}]=\frac{2}{m} \int_{\mathcal{H}(\mathbf{0}, \delta)} \underline{\omega}\langle\boldsymbol{\zeta}\rangle \boldsymbol{\zeta} \cdot(\mathbf{u}(\mathbf{x}+\boldsymbol{\zeta})-\mathbf{u}(\mathbf{x})) d \boldsymbol{\zeta},
$$

where $m$ is the weighted volume,

$$
m:=\int_{\mathcal{H}(\mathbf{0}, \delta)} \underline{\omega}\langle\boldsymbol{\xi}\rangle\|\boldsymbol{\xi}\|^{2} d \boldsymbol{\xi}
$$

155 and $\underline{\omega}\langle\cdot\rangle: \mathbb{R}^{2} \rightarrow \mathbb{R}$ is an influence function. In this paper, we concern ourselves only with radial influence functions, which are those that only depend on the length of bonds. Influence functions are scalar-valued functions determining the support of force states [37 as well as the stiffness of PD bonds [19. Their role in the PD theory has been studied in [19] and their effect on convergence of numerical discretizations has been investigated in $[42,30$.

As in Section 3 , the derivations presented here are for material points $\mathbf{x} \in \mathcal{B}$ in the bulk of the body, so that $\mathcal{H}(\mathbf{x}, \delta) \subset \mathcal{B}$. Expressions for $c_{1}$ and $c_{2}$ can be obtained from the consistency of the PD equilibrium equation (1) to the local limit given, in this case, by the classical Navier-Cauchy equation of static elasticity [28, 29, 46]:

$$
K \frac{\partial^{2} u_{j}}{\partial x_{i} \partial x_{j}}(\mathbf{x})+G \frac{\partial^{2} u_{i}}{\partial x_{k} \partial x_{k}}(\mathbf{x})=-b_{i}(\mathbf{x}),
$$


where $K$ and $G$ are the bulk modulus and shear modulus, respectively. Indeed, we have the following equations satisfied,

$$
\begin{aligned}
& c_{1}=2 K-4 G, \\
& c_{2}=8 G .
\end{aligned}
$$

It can also be shown that the linearized nonlocal dilatation in $(22)$ reduces to the dilatation in classical elasticity, $\nabla \cdot \mathbf{u}$, in the local limit, under proper regularity assumptions (cf. 30]).

Remark 2. In 2D, the elastic moduli satisfy the relations

$$
K=\frac{E}{2(1-\nu)} \quad ; \quad G=\frac{E}{2(1+\nu)}
$$

with $E$ the Young's modulus and $\nu$ the Poisson's ratio 477 . Note that for $\nu=1 / 3$ the

relation $K=2 G$ holds, so that $c_{1}=0$ and (21) effectively reduces to a bond-based PD model.

\subsection{A nearest-neighbor discretization of peridynamic models}

Let a force state be given by (21) with constitutive constants given by $(25)$ and $(26)$. Using the notation $\mathbf{u}=(v, w), \mathbf{x}=(x, y)$, and $\mathbf{b}=\left(b^{1}, b^{2}\right)$, we can express the $\mathrm{PD}$ equilibrium equation (1), in component form, as

$$
\begin{aligned}
& \int_{\mathcal{H}(\mathbf{x}, \delta)}\left\{\frac{2 K-4 G}{m} \omega\left(\left\|\mathbf{x}^{\prime}-\mathbf{x}\right\|\right)\left(x^{\prime}-x\right)\left[\theta^{\operatorname{lin}}[\mathbf{x}]+\theta^{\operatorname{lin}}\left[\mathbf{x}^{\prime}\right]\right]\right. \\
& \left.+\frac{16 G}{m} \frac{\omega\left(\left\|\mathbf{x}^{\prime}-\mathbf{x}\right\|\right)}{\left\|\mathbf{x}^{\prime}-\mathbf{x}\right\|^{2}}\left[\left(x^{\prime}-x\right)^{2}\left(v\left(\mathbf{x}^{\prime}\right)-v(\mathbf{x})\right)+\left(x^{\prime}-x\right)\left(y^{\prime}-y\right)\left(w\left(\mathbf{x}^{\prime}\right)-w(\mathbf{x})\right)\right]\right\} d \mathbf{x}^{\prime} \\
& =-b^{1}(\mathbf{x}) \\
& \int_{\mathcal{H}(\mathbf{x}, \delta)}\left\{\frac{2 K-4 G}{m} \omega\left(\left\|\mathbf{x}^{\prime}-\mathbf{x}\right\|\right)\left(y^{\prime}-y\right)\left[\theta^{\operatorname{lin}}[\mathbf{x}]+\theta^{\operatorname{lin}}\left[\mathbf{x}^{\prime}\right]\right]\right. \\
& \left.+\frac{16 G}{m} \frac{\omega\left(\left\|\mathbf{x}^{\prime}-\mathbf{x}\right\|\right)}{\left\|\mathbf{x}^{\prime}-\mathbf{x}\right\|^{2}}\left[\left(y^{\prime}-y\right)\left(x^{\prime}-x\right)\left(v\left(\mathbf{x}^{\prime}\right)-v(\mathbf{x})\right)+\left(y^{\prime}-y\right)^{2}\left(w\left(\mathbf{x}^{\prime}\right)-w(\mathbf{x})\right)\right]\right\} d \mathbf{x}^{\prime} \\
& =-b^{2}(\mathbf{x})
\end{aligned}
$$

where

$$
\theta^{\operatorname{lin}}[\mathbf{x}]=\frac{2}{m} \int_{\mathcal{H}(\mathbf{x}, \delta)} \omega(\|\hat{\mathbf{x}}-\mathbf{x}\|)[(\hat{x}-x)(v(\hat{\mathbf{x}})-v(\mathbf{x}))+(\hat{y}-y)(w(\hat{\mathbf{x}})-w(\mathbf{x}))] d \hat{\mathbf{x}} .
$$

Let us assume a discretization of a two-dimensional domain using a square grid with grid spacing $h$, and define $x_{i \pm n}:=x_{i} \pm n h$ and $y_{j \pm n}:=y_{j} \pm n h$ with $n \in \mathbb{N}$, $v_{i, j}:=v\left(x_{i}, y_{j}\right), w_{i, j}:=w\left(x_{i}, y_{j}\right), b_{i, j}^{1}:=b^{1}\left(x_{i}, y_{j}\right), b_{i, j}^{2}:=b^{2}\left(x_{i}, y_{j}\right)$, and $\theta_{i, j}^{\operatorname{lin}}:=\theta^{\operatorname{lin}}\left[\mathbf{x}_{i, j}\right]$. 
We denote by $(i, j)$ the node with reference position $\mathbf{x}_{i, j}:=\left(x_{i}, y_{j}\right)$. Using the nodalbased quadrature discretization (4), the discretizations of (28) and (29) at node $(i, j)$ are

$$
\begin{aligned}
& \sum_{(k, \ell) \in \mathcal{F}_{(i, j)}} \frac{\omega\left(\left\|\mathbf{x}_{k, \ell}-\mathbf{x}_{i, j}\right\|\right)}{\left\|\mathbf{x}_{k, \ell}-\mathbf{x}_{i, j}\right\|^{2}}\left\{\frac{2 K-4 G}{m}\left\|\mathbf{x}_{k, \ell}-\mathbf{x}_{i, j}\right\|^{2}\left(x_{k}-x_{i}\right)\left[\theta_{i, j}^{\operatorname{lin}}+\theta_{k, \ell}^{\operatorname{lin}}\right]\right. \\
& \left.\quad+\frac{16 G}{m}\left[\left(x_{k}-x_{i}\right)^{2}\left(v_{k, \ell}-v_{i, j}\right)+\left(x_{k}-x_{i}\right)\left(y_{\ell}-y_{j}\right)\left(w_{k, \ell}-w_{i, j}\right)\right]\right\} V_{k, \ell}^{(i, j)} \\
& =-b_{i, j}^{1}, \\
& \sum_{(k, \ell) \in \mathcal{F}_{(i, j)}} \frac{\omega\left(\left\|\mathbf{x}_{k, \ell}-\mathbf{x}_{i, j}\right\|\right)}{\left\|\mathbf{x}_{k, \ell}-\mathbf{x}_{i, j}\right\|^{2}}\left\{\frac{2 K-4 G}{m}\left\|\mathbf{x}_{k, \ell}-\mathbf{x}_{i, j}\right\|^{2}\left(y_{\ell}-y_{j}\right)\left[\theta_{i, j}^{\operatorname{lin}}+\theta_{k, \ell}^{\operatorname{lin}}\right]\right. \\
& \left.\quad+\frac{16 G}{m}\left[\left(y_{\ell}-y_{j}\right)\left(x_{k}-x_{i}\right)\left(v_{k, \ell}-v_{i, j}\right)+\left(y_{\ell}-y_{j}\right)^{2}\left(w_{k, \ell}-w_{i, j}\right)\right]\right\} V_{k, \ell}^{(i, j)} \\
& =-b_{i, j}^{2},
\end{aligned}
$$

where

$\theta_{i, j}^{\operatorname{lin}}:=\frac{2}{m} \sum_{(p, q) \in \mathcal{F}_{(i, j)}} \omega\left(\left\|\mathbf{x}_{p, q}-\mathbf{x}_{i, j}\right\|\right)\left[\left(x_{p}-x_{i}\right)\left(v_{p, q}-v_{i, j}\right)+\left(y_{q}-y_{j}\right)\left(w_{p, q}-w_{i, j}\right)\right] V_{p, q}^{(i, j)}$,

$V_{k, \ell}^{(i, j)}$ is the quadrature weight associated with the node $(k, \ell)$ in the equation of node $(i, j)$, and $\mathcal{F}_{(i, j)}$ is the family of node $(i, j)$.

Let $A_{L}:=V_{i \pm 1, j}^{(i, j)}=V_{i, j \pm 1}^{(i, j)}$ and $A_{D}:=V_{i \pm 1, j \pm 1}^{(i, j)}$. An illustration is given in Figure 3 We can write a nearest-neighbor discretization of the PD model as follows:

$$
\begin{aligned}
\frac{2 K-4 G}{m} 2 h^{2}[ & \frac{\theta_{i+1, j}^{\operatorname{lin}}-\theta_{i-1, j}^{\operatorname{lin}} \omega(h) A_{L}}{2 h} \\
& \left.+\frac{1}{2}\left(\frac{\theta_{i+1, j+1}^{\operatorname{lin}}-\theta_{i-1, j+1}^{\operatorname{lin}}}{2 h}+\frac{\theta_{i+1, j-1}^{\operatorname{lin}}-\theta_{i-1, j-1}^{\operatorname{lin}}}{2 h}\right) 2 \omega(\sqrt{2} h) A_{D}\right] \\
+\frac{16 G}{m} h^{2}\left[\frac{v_{i+1, j}-2 v_{i, j}+v_{i-1, j}}{h^{2}} \omega(h) A_{L}\right. & {\left[\frac{1}{2} \frac{v_{i+1, j+1}+v_{i+1, j-1}+v_{i-1, j+1}+v_{i-1, j-1}-4 v_{i, j}}{2 h^{2}}\right.} \\
& \left.\left.+\frac{w_{i+1, j+1}-w_{i+1, j-1}-w_{i-1, j+1}+w_{i-1, j-1}}{4 h^{2}}\right) 2 \omega(\sqrt{2} h) A_{D}\right]
\end{aligned}
$$


and

$$
\begin{aligned}
& \frac{2 K-4 G}{m} 2 h^{2}[ \frac{\theta_{i, j+1}^{\operatorname{lin}}-\theta_{i, j-1}^{\operatorname{lin}}}{2 h} \omega(h) A_{L} \\
&\left.+\frac{1}{2}\left(\frac{\theta_{i+1, j+1}^{\operatorname{lin}}-\theta_{i+1, j-1}^{\operatorname{lin}}}{2 h}+\frac{\theta_{i-1, j+1}^{\operatorname{lin}}-\theta_{i-1, j-1}^{\operatorname{lin}}}{2 h}\right) 2 \omega(\sqrt{2} h) A_{D}\right] \\
&+\frac{16 G}{m} h^{2}\left[\frac{w_{i, j+1}-2 w_{i, j}+w_{i, j-1}}{h^{2}} \omega(h) A_{L}\right. \\
&+\left(\frac{1}{2} \frac{w_{i+1, j+1}+w_{i+1, j-1}+w_{i-1, j+1}+w_{i-1, j-1}-4 w_{i, j}}{2 h^{2}}\right. \\
&\left.\left.\quad+\frac{v_{i+1, j+1}-v_{i+1, j-1}-v_{i-1, j+1}+v_{i-1, j-1}}{4 h^{2}}\right) 2 \omega(\sqrt{2} h) A_{D}\right] \\
&=-b_{i, j}^{2},
\end{aligned}
$$

where the discretized linearized nonlocal dilatation is given by

$$
\begin{aligned}
\theta_{i, j}^{\operatorname{lin}}= & \frac{4 h^{2}}{m}\left\{\left[\frac{v_{i+1, j}-v_{i-1, j}}{2 h}+\frac{w_{i, j+1}-w_{i, j-1}}{2 h}\right] \omega(h) A_{L}\right. \\
+ & {\left[\frac{1}{2}\left(\frac{v_{i+1, j+1}-v_{i-1, j+1}}{2 h}+\frac{v_{i+1, j-1}-v_{i-1, j-1}}{2 h}\right)\right.} \\
& \left.\left.+\frac{1}{2}\left(\frac{w_{i+1, j+1}-w_{i+1, j-1}}{2 h}+\frac{w_{i-1, j+1}-w_{i-1, j-1}}{2 h}\right)\right] 2 \omega(\sqrt{2} h) A_{D}\right\} .
\end{aligned}
$$

We now seek quadrature weights $A_{L}$ and $A_{D}$ for which (34) and (35) recover classical local limits. We begin with the discretized linearized nonlocal dilatation (36). Using the following finite difference approximations for first partial derivatives:

$$
\left(\frac{\partial v}{\partial x}\right)_{i, j}=\frac{v_{i+1, j}-v_{i-1, j}}{2 h}+\mathcal{O}\left(h^{2}\right), \quad\left(\frac{\partial v}{\partial y}\right)_{i, j}=\frac{v_{i, j+1}-v_{i, j-1}}{2 h}+\mathcal{O}\left(h^{2}\right),
$$

we obtain, for sufficiently small $h$,

$$
\theta_{i, j}^{\mathrm{lin}}=\frac{4 h^{2}}{m}\left(\omega(h) A_{L}+2 \omega(\sqrt{2} h) A_{D}\right)\left(\left(\frac{\partial v}{\partial x}\right)_{i, j}+\left(\frac{\partial w}{\partial y}\right)_{i, j}\right),
$$

where we have also used the approximations

$$
\begin{aligned}
& \left(\frac{\partial v}{\partial x}\right)_{i, j}=\frac{1}{2}\left(\left(\frac{\partial v}{\partial x}\right)_{i, j+1}+\left(\frac{\partial v}{\partial x}\right)_{i, j-1}\right)+\mathcal{O}\left(h^{2}\right), \\
& \left(\frac{\partial w}{\partial y}\right)_{i, j}=\frac{1}{2}\left(\left(\frac{\partial w}{\partial y}\right)_{i+1, j}+\left(\frac{\partial w}{\partial y}\right)_{i-1, j}\right)+\mathcal{O}\left(h^{2}\right) .
\end{aligned}
$$




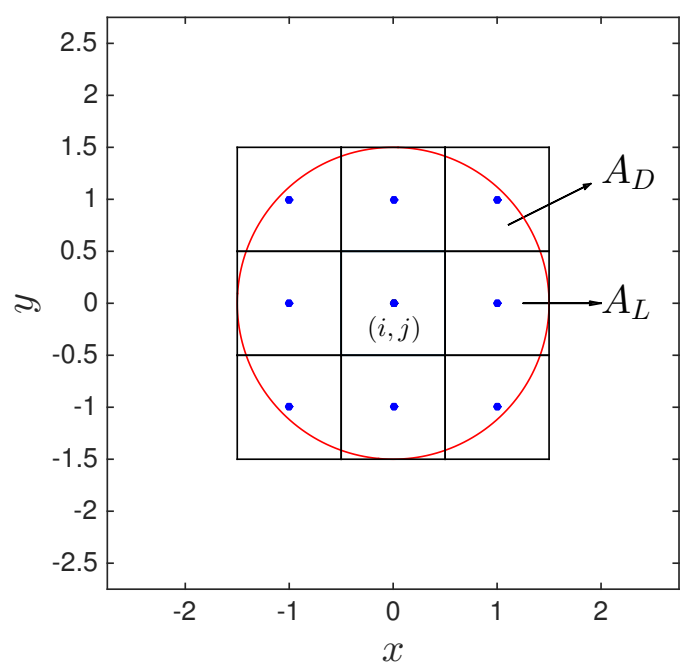

Figure 3: Family of node $(i, j)$ at $\mathbf{x}_{i, j}=(0,0)$ for a nearest-neighbor discretization of a PD system with $\delta=1.5$ and $h=1$. The corresponding quadrature weights $A_{L}$ and $A_{D}$ are indicated.

Using (37), the above finite difference approximations for first partial derivatives, and the following finite difference approximations for second partial derivatives:

$$
\begin{aligned}
\left(\frac{\partial^{2} v}{\partial x^{2}}\right)_{i, j}= & \frac{v_{i+1, j}-2 v_{i, j}+v_{i-1, j}}{h^{2}}+\mathcal{O}\left(h^{2}\right) \\
\left(\frac{\partial^{2} v}{\partial y^{2}}\right)_{i, j}= & \frac{v_{i, j+1}-2 v_{i, j}+v_{i, j-1}}{h^{2}}+\mathcal{O}\left(h^{2}\right) \\
\left(\frac{\partial^{2} v}{\partial x \partial y}\right)_{i, j}= & \frac{v_{i+1, j+1}-v_{i+1, j-1}-v_{i-1, j+1}+v_{i-1, j-1}}{4 h^{2}}+\mathcal{O}\left(h^{2}\right), \\
\left(\frac{\partial^{2} v}{\partial x^{2}}+\frac{\partial^{2} v}{\partial y^{2}}\right)_{i, j}= & \frac{v_{i+1, j+1}+v_{i+1, j-1}+v_{i-1, j+1}+v_{i-1, j-1}-4 v_{i, j}}{2 h^{2}} \\
& +\mathcal{O}\left(h^{2}\right)
\end{aligned}
$$

we obtain from (34) and 35 , respectively, for sufficiently small $h$,

$$
\begin{aligned}
& (K-2 G)\left(\frac{4 h^{2}}{m}\left(\omega(h) A_{L}+2 \omega(\sqrt{2} h) A_{D}\right)\right)^{2}\left(\left(\frac{\partial^{2} v}{\partial x^{2}}\right)_{i, j}+\left(\frac{\partial^{2} w}{\partial x \partial y}\right)_{i, j}\right) \\
& +G\left[4\left(\frac{4 h^{2}}{m} \omega(h) A_{L}\right)\left(\frac{\partial^{2} v}{\partial x^{2}}\right)_{i, j}+2\left(\frac{4 h^{2}}{m} 2 \omega(\sqrt{2} h) A_{D}\right)\left(\frac{\partial^{2} v}{\partial x^{2}}+\frac{\partial^{2} v}{\partial y^{2}}\right)_{i, j}\right. \\
& \left.+4\left(\frac{4 h^{2}}{m} 2 \omega(\sqrt{2} h) A_{D}\right)\left(\frac{\partial^{2} w}{\partial x \partial y}\right)_{i, j}\right]=-b_{i, j}^{1}
\end{aligned}
$$


and

$$
\begin{aligned}
& (K-2 G)\left(\frac{4 h^{2}}{m}\left(\omega(h) A_{L}+2 \omega(\sqrt{2} h) A_{D}\right)\right)^{2}\left(\left(\frac{\partial^{2} w}{\partial y^{2}}\right)_{i, j}+\left(\frac{\partial^{2} v}{\partial x \partial y}\right)_{i, j}\right) \\
& +G\left[4\left(\frac{4 h^{2}}{m} \omega(h) A_{L}\right)\left(\frac{\partial^{2} w}{\partial y^{2}}\right)_{i, j}+2\left(\frac{4 h^{2}}{m} 2 \omega(\sqrt{2} h) A_{D}\right)\left(\frac{\partial^{2} w}{\partial x^{2}}+\frac{\partial^{2} w}{\partial y^{2}}\right)_{i, j}\right. \\
& \left.+4\left(\frac{4 h^{2}}{m} 2 \omega(\sqrt{2} h) A_{D}\right)\left(\frac{\partial^{2} v}{\partial x \partial y}\right)_{i, j}\right]=-b_{i, j}^{2} .
\end{aligned}
$$

Define

$$
\begin{aligned}
B_{L} & :=\frac{4 h^{2}}{m} \omega(h) A_{L}, \\
B_{D} & :=\frac{4 h^{2}}{m} 2 \omega(\sqrt{2} h) A_{D} .
\end{aligned}
$$

Then, we can write (37) as

$$
\theta_{i, j}^{\operatorname{lin}}=\left(B_{L}+B_{D}\right)\left(\left(\frac{\partial v}{\partial x}\right)_{i, j}+\left(\frac{\partial w}{\partial y}\right)_{i, j}\right),
$$

and (39) and 40), respectively, as

$$
\begin{aligned}
& \left(B_{L}+B_{D}\right)^{2} K\left(\left(\frac{\partial^{2} v}{\partial x^{2}}\right)_{i, j}+\left(\frac{\partial^{2} w}{\partial x \partial y}\right)_{i, j}\right)+2\left(2 B_{L}-\left(B_{L}+B_{D}\right)^{2}\right) G\left(\frac{\partial^{2} v}{\partial x^{2}}\right)_{i, j} \\
& +2 B_{D} G\left(\frac{\partial^{2} v}{\partial x^{2}}+\frac{\partial^{2} v}{\partial y^{2}}\right)_{i, j}+2\left(2 B_{D}-\left(B_{L}+B_{D}\right)^{2}\right) G\left(\frac{\partial^{2} w}{\partial x \partial y}\right)_{i, j}=-b_{i, j}^{1}
\end{aligned}
$$

and

$$
\begin{aligned}
& \left(B_{L}+B_{D}\right)^{2} K\left(\left(\frac{\partial^{2} w}{\partial y^{2}}\right)_{i, j}+\left(\frac{\partial^{2} v}{\partial x \partial y}\right)_{i, j}\right)+2\left(2 B_{L}-\left(B_{L}+B_{D}\right)^{2}\right) G\left(\frac{\partial^{2} w}{\partial y^{2}}\right)_{i, j} \\
& +2 B_{D} G\left(\frac{\partial^{2} w}{\partial x^{2}}+\frac{\partial^{2} w}{\partial y^{2}}\right)_{i, j}+2\left(2 B_{D}-\left(B_{L}+B_{D}\right)^{2}\right) G\left(\frac{\partial^{2} v}{\partial x \partial y}\right)_{i, j}=-b_{i, j}^{2}
\end{aligned}
$$

We would like to satisfy the following relations:

$$
\begin{aligned}
B_{L}+B_{D} & =1, \\
2 B_{D} & =1, \\
2 B_{L}-\left(B_{L}+B_{D}\right)^{2} & =0, \\
2 B_{D}-\left(B_{L}+B_{D}\right)^{2} & =0,
\end{aligned}
$$

to recover from 43 the discretized classical dilatation, i.e.,

$$
\theta_{i, j}^{\operatorname{lin}}=\left(\left(\frac{\partial v}{\partial x}\right)_{i, j}+\left(\frac{\partial w}{\partial y}\right)_{i, j}\right),
$$


and to have 44) and 45 reduce to the discretized classical Navier-Cauchy equation of static elasticity in component form, i.e.,

$$
K\left(\left(\frac{\partial^{2} v}{\partial x^{2}}\right)_{i, j}+\left(\frac{\partial^{2} w}{\partial x \partial y}\right)_{i, j}\right)+G\left(\frac{\partial^{2} v}{\partial x^{2}}+\frac{\partial^{2} v}{\partial y^{2}}\right)_{i, j}=-b_{i, j}^{1}
$$

and

$$
K\left(\left(\frac{\partial^{2} w}{\partial y^{2}}\right)_{i, j}+\left(\frac{\partial^{2} v}{\partial x \partial y}\right)_{i, j}\right)+G\left(\frac{\partial^{2} w}{\partial x^{2}}+\frac{\partial^{2} w}{\partial y^{2}}\right)_{i, j}=-b_{i, j}^{2} .
$$

In the following derivations, it is assumed that $\omega(h)>0$ and $\omega(\sqrt{2} h)>0$. By 48 and $(49)$, we obtain

$$
B_{L}=B_{D}
$$

Then, using (41) and (42), we get the following relation between the quadrature weights:

$$
A_{L}=\frac{2 \omega(\sqrt{2} h) A_{D}}{\omega(h)} .
$$

By (47) (or equivalently by (46) with (53)), we obtain

$$
B_{D}=\frac{1}{2}
$$

which results, in combination with (54), in

$$
\begin{aligned}
& A_{D}=\frac{m}{16 \omega(\sqrt{2} h) h^{2}}, \\
& A_{L}=\frac{m}{8 \omega(h) h^{2}} .
\end{aligned}
$$

Let the influence function be $\omega(\|\boldsymbol{\xi}\|)=1 /\|\boldsymbol{\xi}\|^{\alpha}$ with $\alpha<4$. In this case, we get (cf. (23))

$$
m=2 \pi \frac{\delta^{4-\alpha}}{4-\alpha} .
$$

Taking $h=\delta$, we obtain from (56) and (57)

$$
\begin{aligned}
& A_{D}=\frac{(\sqrt{2})^{\alpha} \pi h^{2}}{8(4-\alpha)}, \\
& A_{L}=\frac{\pi h^{2}}{4(4-\alpha)} .
\end{aligned}
$$

In particular, the following relation holds (cf. (54)):

$$
A_{L}=(\sqrt{2})^{2-\alpha} A_{D} .
$$

170 In Table 1 , we present the expressions of $A_{D}$ and $A_{L}$, given by 590 and 60$)$, respectively, for various choices of $\alpha$. 
Table 1: Quadrature weights $A_{D}$ and $A_{L}$ given by 59 and 60 , respectively.

\begin{tabular}{|c|c|c|c|}
\hline & $A_{D}$ & $A_{L}$ & Relation between $A_{L}$ and $A_{D}$ \\
\hline$\alpha=0$ & $\frac{\pi h^{2}}{32}$ & $\frac{\pi h^{2}}{16}$ & $A_{L}=2 A_{D}$ \\
\hline$\alpha=1$ & $\frac{\pi h^{2}}{12 \sqrt{2}}$ & $\frac{\pi h^{2}}{12}$ & $A_{L}=\sqrt{2} A_{D}$ \\
\hline$\alpha=2$ & $\frac{\pi h^{2}}{8}$ & $\frac{\pi h^{2}}{8}$ & $A_{L}=A_{D}$ \\
\hline$\alpha=3$ & $\frac{\sqrt{2} \pi h^{2}}{4}$ & $\frac{\pi h^{2}}{4}$ & $A_{L}=A_{D} / \sqrt{2}$ \\
\hline
\end{tabular}

Remark 3. For a radial influence function of the form $\omega(\|\boldsymbol{\xi}\|)=1 /\|\boldsymbol{\xi}\|^{\alpha}$ with $\alpha=2$ and the choice of grid spacing $h=\delta$, we obtain (cf. Table 1)

$$
A_{L}=A_{D}=\frac{1}{8} \pi \delta^{2} .
$$

This suggests the selection of uniform weights with values of an eighth of the area of the neighborhood of a given node.

We now compare the quadrature weights $(56)$ and (57) with quadrature weights of existing methods:

- Meshfree method: In the meshfree method [20, we have $A_{L}=A_{D}=h^{2}$. By (56) and (57), we obtain $A_{L}=A_{D}$ only if the influence function satisfies $2 \omega(\sqrt{2} h)=\omega(h)$. For the influence function, $\omega(\|\boldsymbol{\xi}\|)=1 /\|\boldsymbol{\xi}\|^{\alpha}$, this is actually satisfied for $\alpha=2$. However, by Table 1, a choice of $\alpha=2$ results in $A_{L}=A_{D}=\pi h^{2} / 8 \neq h^{2}$.

- Partial-volume method:

Let a discretization be given by $h=\delta / \gamma$ with $1 / \sqrt{2}<\gamma \leqslant 3 / 2$. This corresponds to a nearest-neighbor discretization, which includes the closest eight nearest-neighbor cells. An illustration for $\delta=1.5$ and $h=1$ is shown in Figure 3. The quadrature weights $A_{L}$ and $A_{D}$ for the discrete equation of a given node are taken, in this case, as the areas of the intersections between the neighbor cells and the neighborhood of that node 42, 30. Given a value of $\alpha$, we would like to investigate whether there is a value of $\gamma$ such that the conditions $(46)-(49)$ are simultaneously satisfied. For that purpose, we follow [42] and derive analytical expressions for $A_{L}$ and $A_{D}$, given by the corresponding areas, as functions of $\gamma$ ( $c f$. Appendix A $)$. The results are:

$$
\begin{aligned}
& A_{L}=\left[\frac{1}{4}(g(\gamma)-1)+\gamma^{2} \arcsin \left(\frac{1}{2 \gamma}\right)\right] h^{2}, \\
& A_{D}=\left[\frac{1}{8}(g(\gamma))^{2}+\gamma^{2} \arcsin \left(\frac{g(\gamma)}{2 \sqrt{2} \gamma}\right)-\frac{g(\gamma)}{4 \sqrt{2}} \sqrt{(2 \gamma)^{2}-\frac{1}{2}(g(\gamma))^{2}}\right] h^{2},
\end{aligned}
$$

where $g(\gamma):=\sqrt{(2 \gamma)^{2}-1}-1$. In Figure 4, we plot the different factors in 44) and 45 as functions of $\gamma$ for $h=1$, computed with the expressions for $A_{L}$ and $A_{D}$ 
in (62) and (63), respectively, and using the analytical expression for the weighted volume in (58). We observe that if we take the quadrature weights $A_{L}$ and $A_{D}$ as the corresponding intersection areas, there is no value of $\gamma$ for which we simultaneously satisfy the conditions (46)-49) exactly. In the case of $\alpha=0,1$, however, we approximately satisfy those conditions for $\gamma=1.5$, which corresponds to a nearestneighbor discretization with grid spacing $h=\delta / 1.5$.

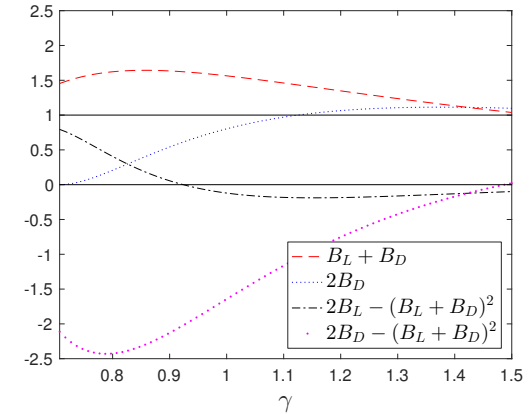

(a) $\alpha=0$

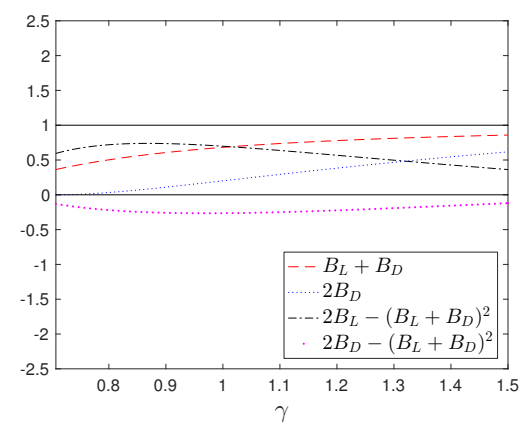

(c) $\alpha=2$

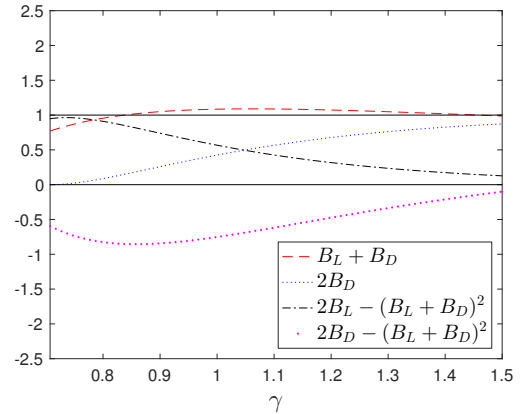

(b) $\alpha=1$

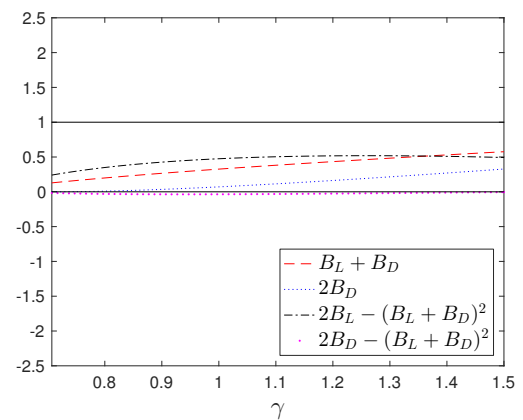

(d) $\alpha=3$

Figure 4: Profiles of factors in 44 and 45 as functions of $\gamma$ for $h=1$, computed with $A_{L}$ and $A_{D}$ in 62 and 63, respectively, and using the weighted volume in 58.

\subsection{Stability analysis}

We perform a Fourier mode analysis to demonstrate the material stability of the discrete scheme given by (34), (35), and (36). For simplicity, we consider the domain $\mathcal{B}=[-\pi, \pi)^{2}$. Given a function $f: \mathcal{B} \rightarrow \mathbb{R}$, its Fourier decomposition can be expressed as

$$
f(x, y)=\sum_{k=-\infty}^{\infty} \sum_{\ell=-\infty}^{\infty} \hat{f}_{k, \ell} e^{\iota(k x+\ell y)}
$$


where $\iota:=\sqrt{-1}$ and $\left\{\hat{f}_{k, \ell}\right\}_{k, \ell=-\infty, \ldots, \infty}$ are the Fourier coefficients. Let the body $\mathcal{B}$ be discretized with a square grid of grid spacing $h$. Then, the evaluation of the function in (64) at $\mathbf{x}_{i, j}=\left(x_{i}, y_{j}\right)$ is

$$
f_{i, j}:=f\left(x_{i}, y_{j}\right)=\sum_{k=-\infty}^{\infty} \sum_{\ell=-\infty}^{\infty} \hat{f}_{k, \ell} e^{\iota\left(k x_{i}+\ell y_{j}\right)} .
$$

Using the Fourier decomposition (65) for the variables in (36) results in the following relation:

$$
\begin{aligned}
\hat{\theta}_{k, \ell}^{\operatorname{lin}}= & \iota B_{L}\left[\hat{v}_{k, \ell} \frac{\sin (k h)}{h}+\hat{w}_{k, \ell} \frac{\sin (\ell h)}{h}\right] \\
& +\iota B_{D}\left[\hat{v}_{k, \ell} \cos (\ell h) \frac{\sin (k h)}{h}+\hat{w}_{k, \ell} \cos (k h) \frac{\sin (\ell h)}{h}\right],
\end{aligned}
$$

where $\hat{\theta}_{k, \ell}^{\operatorname{lin}}$ is a Fourier coefficient for the linearized nonlocal dilatation, and $\hat{v}_{k, \ell}$ and $\hat{w}_{k, \ell}$ are Fourier coefficients for the components of the displacement field: $v$ and $w$, respectively. Taking $B_{L}=B_{D}=1 / 2(c f$. (53) and (55)), we obtain

$$
\hat{\theta}_{k, \ell}^{\operatorname{lin}}=\frac{\iota}{2 h} \sin (k h)[1+\cos (\ell h)] \hat{v}_{k, \ell}+\frac{\iota}{2 h} \sin (\ell h)[1+\cos (k h)] \hat{w}_{k, \ell} .
$$

Using the same Fourier decomposition for the variables in (34) and (35), we obtain, respectively,

$$
\begin{aligned}
& (K-2 G)\left\{\hat{\theta}_{k, \ell}^{\operatorname{lin}} \frac{\iota \sin (k h)}{h}\left[B_{L}+\cos (\ell h) B_{D}\right]\right\} \\
& +4 G\left\{\hat{v}_{k, \ell}\left[2 \frac{\cos (k h)-1}{h^{2}} B_{L}+\frac{\cos (k h) \cos (\ell h)-1}{h^{2}} B_{D}\right]-\hat{w}_{k, \ell} \frac{\sin (k h) \sin (\ell h)}{h^{2}} B_{D}\right\} \\
& =-\hat{b}_{k, \ell}^{1}
\end{aligned}
$$

and

$$
\begin{aligned}
& (K-2 G)\left\{\hat{\theta}_{k, \ell}^{\operatorname{lin}} \frac{\iota \sin (\ell h)}{h}\left[B_{L}+\cos (k h) B_{D}\right]\right\} \\
& +4 G\left\{\hat{w}_{k, \ell}\left[2 \frac{\cos (\ell h)-1}{h^{2}} B_{L}+\frac{\cos (k h) \cos (\ell h)-1}{h^{2}} B_{D}\right]-\hat{v}_{k, \ell} \frac{\sin (k h) \sin (\ell h)}{h^{2}} B_{D}\right\} \\
& =-\hat{b}_{k, \ell}^{2},
\end{aligned}
$$

where $\hat{b}_{k, \ell}^{1}$ and $\hat{b}_{k, \ell}^{2}$ are Fourier coefficients for the components of the body force density field: $b^{1}$ and $b^{2}$, respectively. Taking, as above, $B_{L}=B_{D}=1 / 2$ in 68) and (69), and using (67) and the trigonometric identities

$$
\sin ^{2}(x)=\frac{1-\cos (2 x)}{2} \quad ; \quad \cos ^{2}(x)=\frac{1+\cos (2 x)}{2},
$$


we obtain (after rearranging the equations)

$$
\begin{aligned}
& \frac{K-2 G}{h^{2}}\left\{\sin ^{2}(k h) \cos ^{4}\left(\frac{\ell h}{2}\right) \hat{v}_{k, \ell}+\sin (k h) \sin (\ell h) \cos ^{2}\left(\frac{k h}{2}\right) \cos ^{2}\left(\frac{\ell h}{2}\right) \hat{w}_{k, \ell}\right\} \\
+ & \frac{2 G}{h^{2}}\left\{\left[4 \sin ^{2}\left(\frac{k h}{2}\right)+(1-\cos (k h) \cos (\ell h))\right] \hat{v}_{k, \ell}+\sin (k h) \sin (\ell h) \hat{w}_{k, \ell}\right\}=\hat{b}_{k, \ell}^{1},
\end{aligned}
$$

and

$$
\begin{gathered}
\frac{K-2 G}{h^{2}}\left\{\sin (k h) \sin (\ell h) \cos ^{2}\left(\frac{k h}{2}\right) \cos ^{2}\left(\frac{\ell h}{2}\right) \hat{v}_{k, \ell}+\sin ^{2}(\ell h) \cos ^{4}\left(\frac{k h}{2}\right) \hat{w}_{k, \ell}\right\} \\
+\frac{2 G}{h^{2}}\left\{\sin (k h) \sin (\ell h) \hat{v}_{k, \ell}+\left[4 \sin ^{2}\left(\frac{\ell h}{2}\right)+(1-\cos (k h) \cos (\ell h))\right] \hat{w}_{k, \ell}\right\}=\hat{b}_{k, \ell}^{2} .
\end{gathered}
$$

Given $k, \ell \in \mathbb{Z}$, let $\hat{\mathbf{u}}=\left(\hat{v}_{k, \ell}, \hat{w}_{k, \ell}\right)^{\mathrm{T}}$ and $\hat{\mathbf{b}}=\left(\hat{b}_{k, \ell}^{1}, \hat{b}_{k, \ell}^{2}\right)^{\mathrm{T}}$. Then, the system of equations given by $(70)$ and $(71)$ can be expressed as

$$
\left(\frac{K}{h^{2}} \mathbf{K}+\frac{2 G}{h^{2}} \mathbf{G}\right) \hat{\mathbf{u}}=\hat{\mathbf{b}}
$$

with

$$
\mathbf{K}=\left(\begin{array}{cc}
\sin ^{2}(k h) \cos ^{4}\left(\frac{\ell h}{2}\right) & \sin (k h) \sin (\ell h) \cos ^{2}\left(\frac{k h}{2}\right) \cos ^{2}\left(\frac{\ell h}{2}\right) \\
\sin (k h) \sin (\ell h) \cos ^{2}\left(\frac{k h}{2}\right) \cos ^{2}\left(\frac{\ell h}{2}\right) & \sin ^{2}(\ell h) \cos ^{4}\left(\frac{k h}{2}\right)
\end{array}\right)
$$

and the components of $\mathbf{G}$ given by

$$
\begin{aligned}
& G_{11}=4 \sin ^{2}\left(\frac{k h}{2}\right)+(1-\cos (k h) \cos (\ell h))-\sin ^{2}(k h) \cos ^{4}\left(\frac{\ell h}{2}\right), \\
& G_{12}=G_{21}=\sin (k h) \sin (\ell h)\left(1-\cos ^{2}\left(\frac{k h}{2}\right) \cos ^{2}\left(\frac{\ell h}{2}\right)\right), \\
& G_{22}=4 \sin ^{2}\left(\frac{\ell h}{2}\right)+(1-\cos (k h) \cos (\ell h))-\sin ^{2}(\ell h) \cos ^{4}\left(\frac{k h}{2}\right) .
\end{aligned}
$$

The material stability is implied if the matrices $\mathbf{K}$ and $\mathbf{G}$ are each positive semi-definite. For this purpose, one can employ the principal minor test [48. In the case of a $2 \times 2$ symmetric matrix $\mathbf{A}$, this test requires showing that $A_{11} \geqslant 0$ and $\operatorname{det}(\mathbf{A})=A_{11} A_{22}-$ $A_{12}^{2} \geqslant 0$; these two conditions also imply $A_{22} \geqslant 0$. It is straightforward to show that the matrix $\mathbf{K}$ is positive semidefinite, based on the principal minor test. For the matrix $\mathbf{G}$, let $x=\sin ^{2}\left(\frac{k h}{2}\right)$ and $y=\sin ^{2}\left(\frac{\ell h}{2}\right)$. Then, the following relations hold:

$$
\begin{aligned}
\cos ^{2}\left(\frac{k h}{2}\right) & =1-x \quad ; \quad \cos ^{2}\left(\frac{\ell h}{2}\right)=1-y, \\
\cos (k h) & =1-2 x \quad ; \quad \cos (\ell h)=1-2 y, \\
\sin ^{2}(k h) & =4 x(1-x) \quad ; \quad \sin ^{2}(\ell h)=4 y(1-y) .
\end{aligned}
$$

We can now write

$$
\begin{aligned}
& G_{11}=4 x+(2 x+2 y-4 x y)-4 x(1-x)(1-y)^{2}, \\
& G_{22}=4 y+(2 x+2 y-4 x y)-4 y(1-y)(1-x)^{2}, \\
& G_{12}^{2}=16 x y(1-x)(1-y)(x+y-x y)^{2} .
\end{aligned}
$$

In the derivations below, we use the fact that $(x, y) \in[0,1]^{2}$. We first have

$$
G_{11}=4 x\left(1-(1-x)(1-y)^{2}\right)+2 x(1-y)+2 y(1-x) \geqslant 0
$$


and, similarly, $G_{22} \geqslant 0$. In addition, using the following inequalities:

$$
\begin{aligned}
& 2 x-x y-2 x(1-x)(1-y)^{2}=2 x(1-y)(1-(1-x)(1-y))+x y \geqslant 0, \\
& 2 y-x y-2 y(1-y)(1-x)^{2}=2 y(1-x)(1-(1-y)(1-x))+x y \geqslant 0,
\end{aligned}
$$

we have

$$
\begin{aligned}
\operatorname{det}(\mathbf{G})= & G_{11} G_{22}-G_{12}^{2} \\
= & {\left[4 x+(2 x+2 y-4 x y)-4 x(1-x)(1-y)^{2}\right] } \\
& \times\left[4 y+(2 x+2 y-4 x y)-4 y(1-y)(1-x)^{2}\right] \\
& -16 x y(1-x)(1-y)(x+y-x y)^{2} \\
= & 2\left[2 x-x y-2 x(1-x)(1-y)^{2}+(x+y-x y)\right] \\
& \times 2\left[2 y-x y-2 y(1-y)(1-x)^{2}+(x+y-x y)\right] \\
& -16 x y(1-x)(1-y)(x+y-x y)^{2} \\
\geqslant & 4(x+y-x y)^{2}(1-2 x(1-x) 2 y(1-y)) \geqslant 3(x+y-x y)^{2} \geqslant 0,
\end{aligned}
$$

where the property $2 x(1-x) \leqslant 1 / 2$ for $x \in[0,1]$ has been used. We have thus shown that $\mathbf{G}$ is also positive semi-definite.

\subsection{A quadrature based on a quadratic approximation of the displacement field}

Let a displacement field be given by the following quadratic profile:

$$
\begin{aligned}
& v(x, y)=v_{00}+v_{10} x+v_{01} y+v_{11} x y+v_{20} x^{2}+v_{02} y^{2}, \\
& w(x, y)=w_{00}+w_{10} x+w_{01} y+w_{11} x y+w_{20} x^{2}+w_{02} y^{2},
\end{aligned}
$$

where $v_{00}, v_{10}, v_{01}, v_{11}, v_{20}, v_{02}, w_{00}, w_{10}, w_{01}, w_{11}, w_{20}$, and $w_{02}$ are constant coefficients. Let a body $\mathcal{B}$ be discretized with a square grid of grid spacing $h$. Given a node with reference position $\mathbf{x}_{i, j}=\left(x_{i}, y_{j}\right)$, we have

$$
\begin{aligned}
v_{i, j} & :=v\left(x_{i}, y_{j}\right)=v_{00}+v_{10} x_{i}+v_{01} y_{j}+v_{11} x_{i} y_{j}+v_{20} x_{i}^{2}+v_{02} y_{j}^{2}, \\
w_{i, j} & :=w\left(x_{i}, y_{j}\right)=w_{00}+w_{10} x_{i}+w_{01} y_{j}+w_{11} x_{i} y_{j}+w_{20} x_{i}^{2}+w_{02} y_{j}^{2} .
\end{aligned}
$$

The following basic relations hold:

$$
\begin{aligned}
v_{20} & =\frac{1}{2} \frac{v_{i+1, j}-2 v_{i, j}+v_{i-1, j}}{h^{2}}, \\
v_{02} & =\frac{1}{2} \frac{v_{i, j+1}-2 v_{i, j}+v_{i, j-1}}{h^{2}}, \\
v_{11} & =\frac{v_{i+1, j+1}-v_{i+1, j-1}-v_{i-1, j+1}+v_{i-1, j-1}}{4 h^{2}}, \\
v_{20}+v_{02} & =\frac{v_{i+1, j+1}+v_{i+1, j-1}+v_{i-1, j+1}+v_{i-1, j-1}-4 v_{i, j}}{4 h^{2}},
\end{aligned}
$$

and similarly for the coefficients of $w(x, y)$. Moreover, we have the relations

$$
\begin{aligned}
x^{\prime} y^{\prime}-x y & =\xi_{1} \xi_{2}+y \xi_{1}+x \xi_{2}, \\
\left(x^{\prime}\right)^{2}-x^{2} & =\xi_{1}^{2}+2 x \xi_{1}, \\
\left(y^{\prime}\right)^{2}-y^{2} & =\xi_{2}^{2}+2 y \xi_{2},
\end{aligned}
$$


with $\xi_{1}=x^{\prime}-x$ and $\xi_{2}=y^{\prime}-y$, which allow us to obtain the expressions

$$
\begin{aligned}
v\left(x^{\prime}, y^{\prime}\right)-v(x, y)= & v_{10} \xi_{1}+v_{01} \xi_{2}+v_{11}\left(\xi_{1} \xi_{2}+y \xi_{1}+x \xi_{2}\right)+v_{20}\left(\xi_{1}^{2}+2 x \xi_{1}\right) \\
& +v_{02}\left(\xi_{2}^{2}+2 y \xi_{2}\right) \\
w\left(x^{\prime}, y^{\prime}\right)-w(x, y)= & w_{10} \xi_{1}+w_{01} \xi_{2}+w_{11}\left(\xi_{1} \xi_{2}+y \xi_{1}+x \xi_{2}\right)+w_{20}\left(\xi_{1}^{2}+2 x \xi_{1}\right) \\
& +w_{02}\left(\xi_{2}^{2}+2 y \xi_{2}\right) .
\end{aligned}
$$

Additional relations (in 2D) needed for the derivations below are [44]

$$
\begin{aligned}
\int_{\mathcal{H}(\mathbf{0}, \delta)} \omega(\|\boldsymbol{\xi}\|) \xi_{1}^{2} d \boldsymbol{\xi} & =\int_{\mathcal{H}(\mathbf{0}, \delta)} \omega(\|\boldsymbol{\xi}\|) \xi_{2}^{2} d \boldsymbol{\xi}=\frac{m}{2}, \\
\frac{1}{2} \int_{\mathcal{H}(\mathbf{0}, \delta)} \frac{\omega(\|\boldsymbol{\xi}\|)}{\|\boldsymbol{\xi}\|^{2}} \xi_{i} \xi_{j} \xi_{k} \xi_{\ell} d \boldsymbol{\xi} & =\left(\delta_{i j} \delta_{k \ell}+\delta_{i k} \delta_{j \ell}+\delta_{i \ell} \delta_{j k}\right) \frac{m}{16},
\end{aligned}
$$

with $m$ the weighted volume in 23 .

A direct substitution of (81) and 82 into (30) results in the following expression for the linearized nonlocal dilatation:

$$
\theta^{\operatorname{lin}}[\mathbf{x}]=\left(v_{10}+y v_{11}+2 x v_{20}\right)+\left(w_{01}+x w_{11}+2 y w_{02}\right),
$$

where 83 has been used and antisymmetric terms, which do not contribute to the integration due to the symmetry of the integration domain, have been dropped. In fact, given 73 and $(74)$, the result in 85 ) corresponds to the classical dilatation, i.e.,

$$
\theta^{\operatorname{lin}}[\mathbf{x}]=\frac{\partial v}{\partial x}(x, y)+\frac{\partial w}{\partial y}(x, y)=\nabla \cdot \mathbf{u}(\mathbf{x}) .
$$

By (85), the discretized linearized nonlocal dilatation at a node with reference position $\mathbf{x}_{i, j}=\left(x_{i}, y_{j}\right)$ can be written as

$$
\theta_{i, j}^{\operatorname{lin}}=\left(v_{10}+y_{j} v_{11}+2 x_{i} v_{20}\right)+\left(w_{01}+x_{i} w_{11}+2 y_{j} w_{02}\right) .
$$

We now demonstrate that for a quadratic approximation of the displacement field (given by $(73)$ and (74) ) the nearest-neighbor discretization of the linearized nonlocal dilatation in (36) recovers (86) under a proper choice of quadrature weights. A direct substitution of 75 and 76 into that equation gives

$$
\theta_{i, j}^{\operatorname{lin}}=\left[\left(v_{10}+y_{j} v_{11}+2 x_{i} v_{20}\right)+\left(w_{01}+x_{i} w_{11}+2 y_{j} w_{02}\right)\right]\left(\frac{4 h^{2}}{m} \omega(h) A_{L}+\frac{8 h^{2}}{m} \omega(\sqrt{2} h) A_{D}\right) .
$$

To recover (86), the quadrature weights have to satisfy the relation

$$
\frac{4 h^{2}}{m} \omega(h) A_{L}+\frac{8 h^{2}}{m} \omega(\sqrt{2} h) A_{D}=1,
$$

resulting in the expressions

$$
\begin{aligned}
& A_{D}=\beta \frac{m}{8 h^{2} \omega(\sqrt{2} h)}, \\
& A_{L}=(1-\beta) \frac{m}{4 h^{2} \omega(h)} .
\end{aligned}
$$


Choosing $\beta=1 / 2$, we recover (56) and (57).

We now look at the PD equilibrium equation in component form. By (28) and (29), using (81)-85), we obtain

$$
\begin{aligned}
& -b^{1}(\mathbf{x})=(K-2 G)\left(2 v_{20}+w_{11}\right)+2 G\left(3 v_{20}+v_{02}+w_{11}\right), \\
& -b^{2}(\mathbf{x})=(K-2 G)\left(v_{11}+2 w_{02}\right)+2 G\left(v_{11}+w_{20}+3 w_{02}\right) .
\end{aligned}
$$

These equations can, in fact, be written as

$$
\begin{aligned}
& -b^{1}(\mathbf{x})=(K-2 G)\left(\frac{\partial^{2} v}{\partial x^{2}}(\mathbf{x})+\frac{\partial^{2} w}{\partial x \partial y}(\mathbf{x})\right)+G\left(3 \frac{\partial^{2} v}{\partial x^{2}}(\mathbf{x})+\frac{\partial^{2} v}{\partial y^{2}}(\mathbf{x})+2 \frac{\partial^{2} w}{\partial x \partial y}(\mathbf{x})\right), \\
& -b^{2}(\mathbf{x})=(K-2 G)\left(\frac{\partial^{2} v}{\partial x \partial y}(\mathbf{x})+\frac{\partial^{2} w}{\partial y^{2}}(\mathbf{x})\right)+G\left(2 \frac{\partial^{2} v}{\partial x \partial y}(\mathbf{x})+\frac{\partial^{2} w}{\partial x^{2}}(\mathbf{x})+3 \frac{\partial^{2} w}{\partial y^{2}}(\mathbf{x})\right),
\end{aligned}
$$

which correspond to the classical Navier-Cauchy equation of static elasticity in component form (cf. (24)). Given the nearest-neighbor discretization of the PD model in (34) and (35), a direct substitution of (75), (76), and (86) into those equations gives

$$
\begin{aligned}
-b_{i, j}^{1}= & (K-2 G)\left[\left(2 v_{20}+w_{11}\right)\left(\frac{4 h^{2} \omega(h)}{m} A_{L}+\frac{8 h^{2} \omega(\sqrt{2} h)}{m} A_{D}\right)\right] \\
& +2 G\left[2 v_{20} \frac{8 h^{2} \omega(h)}{m} A_{L}+\left(v_{20}+v_{02}+w_{11}\right) \frac{16 h^{2} \omega(\sqrt{2} h)}{m} A_{D}\right], \\
-b_{i, j}^{2}=( & K-2 G)\left[\left(v_{11}+2 w_{02}\right)\left(\frac{4 h^{2} \omega(h)}{m} A_{L}+\frac{8 h^{2} \omega(\sqrt{2} h)}{m} A_{D}\right)\right] \\
& +2 G\left[2 w_{02} \frac{8 h^{2} \omega(h)}{m} A_{L}+\left(v_{11}+w_{20}+w_{02}\right) \frac{16 h^{2} \omega(\sqrt{2} h)}{m} A_{D}\right],
\end{aligned}
$$

where we have used $(77)-(80)$. To recover $(90)$ and $(91)$, the quadrature weights $A_{D}$ and $A_{L}$ have to be given by (56) and (57), respectively.

Remark 4. For a nearest-neighbor discretization, the dilatational component of the PD model does not require the use of nodes in diagonal positions within the family of a given node (cf. Figure 3). This can be observed by noting that (87) holds for a choice of $\beta=0$ (cf. 888 and (89)). On the contrary, the shear component of the PD model requires both $A_{D}$ and $A_{L}$ to be positive and, in particular, to have values given by (56) and (57), respectively.

4.4. An approach based on a discrete normalization of peridynamic constitutive constants

In Section 3.3. we demonstrated that in a one-dimensional linear elastic bond-based PD model, a normalization of the constitutive constant at the discrete level results in that a nearest-neighbor discretization of the PD model recovers a corresponding discrete local equation. We now investigate whether a similar normalization approach could result 
215 in that a nearest-neighbor discretization of the linear elastic state-based PD model with force state given by (21) reduces as well to a discretization of a corresponding classical local model, regardless of the choice of quadrature weights $A_{D}$ and $A_{L}$.

Let us assume a square grid with grid spacing $h=\delta / \gamma$ with $1 / \sqrt{2}<\gamma \leqslant 3 / 2$, which corresponds to a nearest-neighbor discretization ( $c f$. Figure 3). Computing the weighted volume (23) using the same nodal-based quadrature as that for the PD model, we obtain

$$
m=4 h^{2}\left(\omega(h) A_{L}+2 \omega(\sqrt{2} h) A_{D}\right) .
$$

For a sufficiently small $h$, by (37),

$$
\theta_{i, j}^{\operatorname{lin}}=\left(\frac{\partial v}{\partial x}\right)_{i, j}+\left(\frac{\partial w}{\partial y}\right)_{i, j},
$$

i.e., a discrete approximation of the linearized nonlocal dilatation, based on a nearestneighbor discretization, reduces to a discrete approximation of the classical dilatation, regardless of the choice of $A_{D}$ and $A_{L}$.

We then consider a PD model given by the force state (21) with general constants $c_{1}$ and $c_{2}$, not necessarily given by 25 and (26), respectively. Assuming a quadratic displacement field and employing Taylor expansions, we can write the PD equilibrium equation (1) in component form (cf. (28) and (29) as

$$
\begin{aligned}
& {\left[\frac{c_{1}}{m}\left(\int_{\mathcal{H}(\mathbf{0}, \delta)} \omega(\|\boldsymbol{\xi}\|) \xi_{1}^{2} d \boldsymbol{\xi}\right)+2 \frac{c_{2}}{m}\left(\int_{\mathcal{H}(\mathbf{0}, \delta)} \omega(\|\boldsymbol{\xi}\|) \frac{\xi_{1}^{2} \xi_{2}^{2}}{\|\boldsymbol{\xi}\|^{2}} d \boldsymbol{\xi}\right)\right]\left(\frac{\partial^{2} v}{\partial x^{2}}(\mathbf{x})+\frac{\partial^{2} w}{\partial x \partial y}(\mathbf{x})\right)} \\
& +\frac{c_{2}}{m}\left(\int_{\mathcal{H}(\mathbf{0}, \delta)} \omega(\|\boldsymbol{\xi}\|) \frac{\xi_{1}^{2} \xi_{2}^{2}}{\|\boldsymbol{\xi}\|^{2}} d \boldsymbol{\xi}\right)\left(\frac{\partial^{2} v}{\partial x^{2}}(\mathbf{x})+\frac{\partial^{2} v}{\partial y^{2}}(\mathbf{x})\right) \\
& +\frac{c_{2}}{m}\left[\left(\int_{\mathcal{H}(\mathbf{0}, \delta)} \omega(\|\boldsymbol{\xi}\|) \xi_{1}^{2} d \boldsymbol{\xi}-4 \int_{\mathcal{H}(\mathbf{0}, \delta)} \omega(\|\boldsymbol{\xi}\|) \frac{\xi_{1}^{2} \xi_{2}^{2}}{\|\boldsymbol{\xi}\|^{2}} d \boldsymbol{\xi}\right) \frac{\partial^{2} v}{\partial x^{2}}(\mathbf{x})\right]=-b^{1}(\mathbf{x})
\end{aligned}
$$

and

$$
\begin{aligned}
& {\left[\frac{c_{1}}{m}\left(\int_{\mathcal{H}(\mathbf{0}, \delta)} \omega(\|\boldsymbol{\xi}\|) \xi_{2}^{2} d \boldsymbol{\xi}\right)+2 \frac{c_{2}}{m}\left(\int_{\mathcal{H}(\mathbf{0}, \delta)} \omega(\|\boldsymbol{\xi}\|) \frac{\xi_{1}^{2} \xi_{2}^{2}}{\|\boldsymbol{\xi}\|^{2}} d \boldsymbol{\xi}\right)\right]\left(\frac{\partial^{2} w}{\partial y^{2}}(\mathbf{x})+\frac{\partial^{2} v}{\partial x \partial y}(\mathbf{x})\right)} \\
& +\frac{c_{2}}{m}\left(\int_{\mathcal{H}(\mathbf{0}, \delta)} \omega(\|\boldsymbol{\xi}\|) \frac{\xi_{1}^{2} \xi_{2}^{2}}{\|\boldsymbol{\xi}\|^{2}} d \boldsymbol{\xi}\right)\left(\frac{\partial^{2} w}{\partial x^{2}}(\mathbf{x})+\frac{\partial^{2} w}{\partial y^{2}}(\mathbf{x})\right) \\
& +\frac{c_{2}}{m}\left[\left(\int_{\mathcal{H}(\mathbf{0}, \delta)} \omega(\|\boldsymbol{\xi}\|) \xi_{2}^{2} d \boldsymbol{\xi}-4 \int_{\mathcal{H}(\mathbf{0}, \delta)} \omega(\|\boldsymbol{\xi}\|) \frac{\xi_{1}^{2} \xi_{2}^{2}}{\|\boldsymbol{\xi}\|^{2}} d \boldsymbol{\xi}\right) \frac{\partial^{2} w}{\partial y^{2}}(\mathbf{x})\right]=-b^{2}(\mathbf{x}),
\end{aligned}
$$

where the following identities have been used:

$$
\begin{aligned}
\int_{\mathcal{H}(\mathbf{0}, \delta)} \omega(\|\boldsymbol{\xi}\|) \frac{\xi_{1}^{4}}{\|\boldsymbol{\xi}\|^{2}} d \boldsymbol{\xi}=\int_{\mathcal{H}(\mathbf{0}, \delta)} \omega(\|\boldsymbol{\xi}\|) \xi_{1}^{2} d \boldsymbol{\xi}-\int_{\mathcal{H}(\mathbf{0}, \delta)} \omega(\|\boldsymbol{\xi}\|) \frac{\xi_{1}^{2} \xi_{2}^{2}}{\|\boldsymbol{\xi}\|^{2}} d \boldsymbol{\xi} \\
\int_{\mathcal{H}(\mathbf{0}, \delta)} \omega(\|\boldsymbol{\xi}\|) \frac{\xi_{2}^{4}}{\|\boldsymbol{\xi}\|^{2}} d \boldsymbol{\xi}=\int_{\mathcal{H}(\mathbf{0}, \delta)} \omega(\|\boldsymbol{\xi}\|) \xi_{2}^{2} d \boldsymbol{\xi}-\int_{\mathcal{H}(\mathbf{0}, \delta)} \omega(\|\boldsymbol{\xi}\|) \frac{\xi_{1}^{2} \xi_{2}^{2}}{\|\boldsymbol{\xi}\|^{2}} d \boldsymbol{\xi} .
\end{aligned}
$$


By choosing

$$
\begin{aligned}
& c_{2}=G \frac{m}{\int_{\mathcal{H}(\mathbf{0}, \delta)} \omega(\|\boldsymbol{\xi}\|) \frac{\xi_{1}^{2} \xi_{2}^{2}}{\|\boldsymbol{\xi}\|^{2}} d \boldsymbol{\xi}}, \\
& c_{1}=(K-2 G) \frac{m}{\int_{\mathcal{H}(\mathbf{0}, \delta)} \omega(\|\boldsymbol{\xi}\|) \xi_{1}^{2} d \boldsymbol{\xi}}=(K-2 G) \frac{m}{\int_{\mathcal{H}(\mathbf{0}, \delta)} \omega(\|\boldsymbol{\xi}\|) \xi_{2}^{2} d \boldsymbol{\xi}},
\end{aligned}
$$

we obtain

$$
\begin{aligned}
& K\left(\frac{\partial^{2} v}{\partial x^{2}}(\mathbf{x})+\frac{\partial^{2} w}{\partial x \partial y}(\mathbf{x})\right)+G\left(\frac{\partial^{2} v}{\partial x^{2}}(\mathbf{x})+\frac{\partial^{2} v}{\partial y^{2}}(\mathbf{x})\right)+G\left(\frac{\int_{\mathcal{H}(\mathbf{0}, \delta)} \omega(\|\boldsymbol{\xi}\|) \xi_{1}^{2} d \boldsymbol{\xi}}{\int_{\mathcal{H}(\mathbf{0}, \delta)} \omega(\|\boldsymbol{\xi}\|) \frac{\xi_{1}^{2} \xi_{2}^{2}}{\|\boldsymbol{\xi}\|^{2}} d \boldsymbol{\xi}}-4\right) \frac{\partial^{2} v}{\partial x^{2}}(\mathbf{x}) \\
& =-b^{1}(\mathbf{x})
\end{aligned}
$$

and

$$
\begin{aligned}
& K\left(\frac{\partial^{2} w}{\partial y^{2}}(\mathbf{x})+\frac{\partial^{2} v}{\partial x \partial y}(\mathbf{x})\right)+G\left(\frac{\partial^{2} w}{\partial x^{2}}(\mathbf{x})+\frac{\partial^{2} w}{\partial y^{2}}(\mathbf{x})\right)+G\left(\frac{\int_{\mathcal{H}(\mathbf{0}, \delta)} \omega(\|\boldsymbol{\xi}\|) \xi_{2}^{2} d \boldsymbol{\xi}}{\int_{\mathcal{H}(\mathbf{0}, \delta)} \omega(\|\boldsymbol{\xi}\|) \frac{\xi_{1}^{2} \xi_{2}^{2}}{\|\boldsymbol{\xi}\|^{2}} d \boldsymbol{\xi}}-4\right) \frac{\partial^{2} w}{\partial y^{2}}(\mathbf{x}) \\
& =-b^{2}(\mathbf{x})
\end{aligned}
$$

The following relation holds:

$$
\int_{\mathcal{H}(\mathbf{0}, \delta)} \omega(\|\boldsymbol{\xi}\|) \xi_{1}^{2} d \boldsymbol{\xi}=\int_{\mathcal{H}(\mathbf{0}, \delta)} \omega(\|\boldsymbol{\xi}\|) \xi_{2}^{2} d \boldsymbol{\xi}=4 \int_{\mathcal{H}(\mathbf{0}, \delta)} \omega(\|\boldsymbol{\xi}\|) \frac{\xi_{1}^{2} \xi_{2}^{2}}{\|\boldsymbol{\xi}\|^{2}} d \boldsymbol{\xi}
$$

Consequently, $(99)$ and 100 reduce to the components of the classical Navier-Cauchy equation of static elasticity ( $c f$. (51) and (52)). For a nearest-neighbor discretization, using the same nodal-based quadrature as the one used in the discretization of the governing equations, we have ( $c f .(96))$

$$
\begin{aligned}
\int_{\mathcal{H}(\mathbf{0}, \delta)} \omega(\|\boldsymbol{\xi}\|) \xi_{1}^{2} d \boldsymbol{\xi} & =\int_{\mathcal{H}(\mathbf{0}, \delta)} \omega(\|\boldsymbol{\xi}\|) \xi_{2}^{2} d \boldsymbol{\xi}=2 \omega(h) h^{2} A_{L}+4 \omega(\sqrt{2} h) h^{2} A_{D}=\frac{m}{2}, \\
\int_{\mathcal{H}(\mathbf{0}, \delta)} \omega(\|\boldsymbol{\xi}\|) \frac{\xi_{1}^{2} \xi_{2}^{2}}{\|\boldsymbol{\xi}\|^{2}} d \boldsymbol{\xi} & =2 \omega(\sqrt{2} h) h^{2} A_{D} .
\end{aligned}
$$

Then, we obtain from (97) and (98), respectively,

$$
\begin{aligned}
& c_{2}=G \frac{m}{2 \omega(\sqrt{2} h) h^{2} A_{D}}, \\
& c_{1}=2 K-4 G .
\end{aligned}
$$


We can thus write the nearest-neighbor discretization of the PD equilibrium equation in component form ( $c f .(39)$ and (40)), but with the generalized constants $c_{1}$ and $c_{2}$ given by (102) and (101), respectively, as

$$
\begin{aligned}
& (K-2 G)\left(\left(\frac{\partial^{2} v}{\partial x^{2}}\right)_{i, j}+\left(\frac{\partial^{2} w}{\partial x \partial y}\right)_{i, j}\right) \\
& +G\left[\left(1+2 \frac{\omega(h) A_{L}}{2 \omega(\sqrt{2} h) A_{D}}\right)\left(\frac{\partial^{2} v}{\partial x^{2}}\right)_{i, j}+\left(\frac{\partial^{2} v}{\partial y^{2}}\right)_{i, j}+2\left(\frac{\partial^{2} w}{\partial x \partial y}\right)_{i, j}\right]=-b_{i, j}^{1}
\end{aligned}
$$

and

$$
\begin{aligned}
& (K-2 G)\left(\left(\frac{\partial^{2} w}{\partial y^{2}}\right)_{i, j}+\left(\frac{\partial^{2} v}{\partial x \partial y}\right)_{i, j}\right) \\
& +G\left[\left(1+2 \frac{\omega(h) A_{L}}{2 \omega(\sqrt{2} h) A_{D}}\right)\left(\frac{\partial^{2} w}{\partial y^{2}}\right)_{i, j}+\left(\frac{\partial^{2} w}{\partial x^{2}}\right)_{i, j}+2\left(\frac{\partial^{2} v}{\partial x \partial y}\right)_{i, j}\right]=-b_{i, j}^{2} .
\end{aligned}
$$

To have 103) and (104) reduce, respectively, to discretizations of (92) and (93), the relation between $A_{D}$ and $A_{L}$ given by (54) is required. With that relation, $c_{2}$ in (101) also reduces to (26).

We observe that, in general, a normalization of constitutive constants at the discrete level is not enough to obtain the desired consistency.

\section{Concluding remarks}

It has been demonstrated in the literature that, at the continuum level, the classical elasticity theory can be presented as a limiting case of the peridynamic (PD) theory of solid mechanics. Establishing such a relation at the discrete level is a much more subtle issue. In fact, it has been shown in the literature that discretizations of one-dimensional linear elastic PD equations may or may not converge to classical counterparts based on some popular schemes [31, while in the multidimensional space setting such studies have been mostly limited to finite element methods 32 .

In this paper, we explored connections between nearest-neighbor discretizations of PD equilibrium equations, using nodal-based quadratures, and finite difference discretizations of the classical Navier-Cauchy equation of static elasticity. The meshfree approach commonly used in PD simulations is a special case of the PD discretizations studied in this paper. Our studies included one-dimensional linear elastic bond-based PD models and two-dimensional linear elastic state-based PD models. In 1D, we showed that nearestneighbor discretizations of PD equations only reduce, in general, to classical discrete ones when certain model-/discretization-dependent quadrature weights are employed, or when constitutive constants are normalized at the discrete level. In 2D, we demonstrated that it is also possible to choose particular quadrature weights that lead to the fact that nearest-neighbor discretizations of PD models reduce to their classical discrete counterparts. However, we also demonstrated that it is not possible, in general, to achieve the same result by only normalizing constitutive constants at the discrete level, as opposed to the results obtained in one dimension. Analysis in one dimension also indicated that 
the asymptotic behavior of PD force states may suggest specific quadrature rules. As an example, depending on the singularity of the integrand in the PD internal force density, one may choose either a rectangle method, a trapezoidal rule, or the Simpson's rule for numerical integration. Both in $1 \mathrm{D}$ and $2 \mathrm{D}$, the choice of quadrature weights for nearestneighbor discretizations of PD models, which are consistent with classical (local) discrete equations, can be motivated by a quadratic approximation of displacement fields. This seems natural because PD and classical static problems based on the models studied in this paper share (at the continuum level) the same quadratic solutions, given the same body force density field and consistent boundary conditions.

The implications of this paper are twofold. First, results demonstrated that it may not be possible, without taking additional precautions, to naively take available PD software and run simulations based on nearest-neighbor discretizations to recover numerical results from classical continuum mechanics. Attempting to do that, may lead, in general, to wrong results. Second, straightforward meshfree PD simulations seem to not only be limited on how coarse the computational grid can be chosen (due to the restriction that for $h>\delta$ computational nodes become disconnected), but also be, in general, inaccurate for a choice of $h \approx \delta$. More sophisticated simulation schemes have to be developed to deal with these particular situations.

Nearest-neighbor discretizations of PD models mostly employ a horizon-to-grid spacing ratio, $\delta / h$, somewhere between 1 and 1.5 . It has been numerically observed in simulations involving cracks that a minimum $\delta / h$ ratio is required to prevent grid-dependent crack paths [49, 50, 19, 51] or even formation of spurious microcracks [52]. A larger

${ }_{270} \delta / h$ ratio has thus been recommended for practical PD simulations [53, 15, 24. This manuscript studied a complementary aspect of nearest-neighbor discretizations of PD models and demonstrated that such discretizations are, in general, problematic under the current practice of meshfree PD simulations, because of lack of consistency with classical discrete equations. Our goal was to analytically explore nearest-neighbor dis275 cretizations of PD models, compare them against classical discrete equations, and offer modifications to make them consistent with each other.

The results in this paper provide insights for more robust discretizations of PD models, in particular, for those based upon nodal-based integrations, which are related to particlebased descriptions of PD systems.

\section{Acknowledgements}

The work of P. Seleson was supported in part by the Householder Fellowship which is jointly funded by: the U.S. Department of Energy, Office of Science, Office of Advanced Scientific Computing Research, Applied Mathematics program, under award number ERKJE45, and the Laboratory Directed Research and Development program at the Oak

285 Ridge National Laboratory (ORNL), which is operated by UT-Battelle, LLC., for the U.S. Department of Energy under Contract DE-AC05-00OR22725. The work of Q. Du was supported in part by the U.S. NSF grant DMS-1318586, and the AFOSR MURI center for material failure prediction through peridynamics. Sandia National Laboratories is a multi-program laboratory managed and operated by Sandia Corporation, a wholly 290 owned subsidiary of Lockheed Martin Corporation, for the U.S. Department of Energy's National Nuclear Security Administration under contract DE-AC04-94AL85000. The first author would also like to acknowledge helpful discussions with Konrad Genser and 
Yohan John. Finally, we would like to thank three anonymous referees for their helpful comments and insights.

\section{Appendix A. Calculation of $A_{L}$ and $A_{D}$ as intersecting areas}

In this appendix, we derive analytical expressions for the quadrature weights $A_{L}$ and $A_{D}$ given by corresponding intersecting areas ( $c f$. Figure 3), following [42]. We assume a discretization of a two-dimensional domain given by a square grid with grid spacing $h$.

We begin by calculating $A_{L}$, illustrated as a gray region in Figure A.5. which represents the intersection between the neighborhood of a node at $\mathbf{x}_{i, j}=\left(x_{i}, y_{j}\right)$ and a neighbor cell centered at $\mathbf{x}_{i+1, j}=\left(x_{i}+h, y_{j}\right)$. We compute first the following quantities:

$$
\begin{aligned}
l & =\sqrt{\delta^{2}-\left(\frac{h}{2}\right)^{2}}, \\
\varphi & =\arcsin \left(\frac{h / 2}{\delta}\right) .
\end{aligned}
$$

The area $A_{L}$ can be decomposed into two areas: $A_{1}$ and $A_{2}$, computed as

$$
\begin{aligned}
& A_{1}=\left(l-\frac{h}{2}\right) h, \\
& A_{2}=\varphi \delta^{2}-\frac{1}{2} h l .
\end{aligned}
$$

Let a nearest-neighbor discretization be given by $h=\delta / \gamma$ with $1 / \sqrt{2}<\gamma \leqslant 3 / 2$. Then, we obtain

$$
A_{L}=A_{1}+A_{2}=\left[\frac{1}{4} \sqrt{(2 \gamma)^{2}-1}-\frac{1}{2}+\gamma^{2} \arcsin \left(\frac{1}{2 \gamma}\right)\right] h^{2} .
$$

Define $g(\gamma):=\sqrt{(2 \gamma)^{2}-1}-1$. Then, we can write

$$
A_{L}=\left[\frac{1}{4}(g(\gamma)-1)+\gamma^{2} \arcsin \left(\frac{1}{2 \gamma}\right)\right] h^{2} .
$$

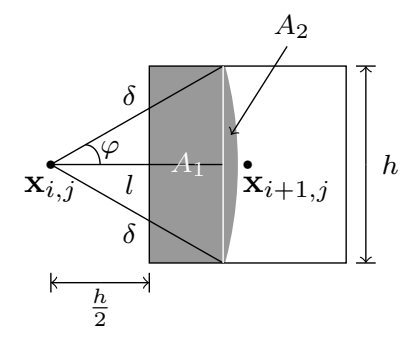

Figure A.5: Geometry decomposition for $A_{L}$. 


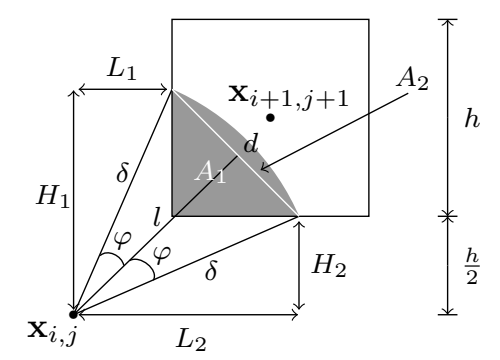

Figure A.6: Geometry decomposition for $A_{D}$.

We now calculate $A_{D}$, illustrated as a gray region in Figure A.6, which represents the intersection between the neighborhood of a node at $\mathbf{x}_{i, j}=\left(x_{i}, y_{j}\right)$ and a neighbor cell centered at $\mathbf{x}_{i+1, j+1}=\left(x_{i}+h, y_{j}+h\right)$. We compute first the following quantities:

$$
\begin{aligned}
L_{1} & =H_{2}=\frac{h}{2} \\
H_{1} & =L_{2}=\sqrt{\delta^{2}-\left(\frac{h}{2}\right)^{2}}, \\
d & =\sqrt{\left(L_{2}-L_{1}\right)^{2}+\left(H_{1}-H_{2}\right)^{2}}=\sqrt{2}\left[\sqrt{\delta^{2}-\left(\frac{h}{2}\right)^{2}}-\frac{h}{2}\right], \\
l & =\sqrt{\delta^{2}-\left(\frac{d}{2}\right)^{2}}=\sqrt{\delta^{2}-\frac{1}{2}\left[\sqrt{\delta^{2}-\left(\frac{h}{2}\right)^{2}}-\frac{h}{2}\right]^{2}}, \\
\varphi & =\arcsin \left(\frac{d / 2}{\delta}\right)=\arcsin \left(\frac{1}{\sqrt{2} \delta}\left[\sqrt{\delta^{2}-\left(\frac{h}{2}\right)^{2}}-\frac{h}{2}\right]\right) .
\end{aligned}
$$

The area $A_{D}$ can be decomposed into two areas: $A_{1}$ and $A_{2}$, computed as

$$
\begin{aligned}
A_{1}=\frac{1}{2}\left(L_{2}-L_{1}\right)\left(H_{1}-H_{2}\right)=\frac{1}{2}\left(\sqrt{\delta^{2}-\left(\frac{h}{2}\right)^{2}}-\frac{h}{2}\right)^{2} \\
\begin{aligned}
A_{2}=\varphi \delta^{2}-\frac{1}{2} d l= & \arcsin \left(\frac{1}{\sqrt{2} \delta}\left[\sqrt{\delta^{2}-\left(\frac{h}{2}\right)^{2}}-\frac{h}{2}\right]\right) \delta^{2} \\
& -\frac{1}{2} \sqrt{2}\left[\sqrt{\delta^{2}-\left(\frac{h}{2}\right)^{2}}-\frac{h}{2}\right] \sqrt{\delta^{2}-\frac{1}{2}\left[\sqrt{\delta^{2}-\left(\frac{h}{2}\right)^{2}}-\frac{h}{2}\right]^{2}} .
\end{aligned}
\end{aligned}
$$

Let a nearest-neighbor discretization be given by $h=\delta / \gamma$ with $1 / \sqrt{2}<\gamma \leqslant 3 / 2$. Then, we obtain

$$
\begin{aligned}
A_{D}=A_{1}+A_{2}= & \frac{1}{8}\left(\sqrt{(2 \gamma)^{2}-1}-1\right)^{2}+\gamma^{2} \arcsin \left(\frac{1}{2 \sqrt{2} \gamma}\left[\sqrt{(2 \gamma)^{2}-1}-1\right]\right) \\
& \left.-\frac{1}{4 \sqrt{2}}\left[\sqrt{(2 \gamma)^{2}-1}-1\right] \sqrt{(2 \gamma)^{2}-\frac{1}{2}\left[\sqrt{(2 \gamma)^{2}-1}-1\right]^{2}}\right] h^{2}
\end{aligned}
$$


This can be written as

$$
A_{D}=\left[\frac{1}{8}(g(\gamma))^{2}+\gamma^{2} \arcsin \left(\frac{g(\gamma)}{2 \sqrt{2} \gamma}\right)-\frac{g(\gamma)}{4 \sqrt{2}} \sqrt{(2 \gamma)^{2}-\frac{1}{2}(g(\gamma))^{2}}\right] h^{2}
$$

300 where $g(\gamma)$ is defined above.

\section{References}

[1] S. A. Silling, Reformulation of elasticity theory for discontinuities and long-range forces, J. Mech. Phys. Solids 48 (2000) 175-209.

[2] P. Seleson, M. L. Parks, M. Gunzburger, R. B. Lehoucq, Peridynamics as an upscaling of molecular dynamics, Multiscale Model. Simul. 8 (1) (2009) 204-227.

[3] P. Seleson, M. L. Parks, M. Gunzburger, Peridynamic state-based models and the embedded-atom model, Commun. Comput. Phys. 15 (1) (2014) 179-205.

[4] Y. D. Ha, F. Bobaru, Studies of dynamic crack propagation and crack branching with peridynamics, Int J. Fract. 162 (2010) 229-244.

310 [5] S. A. Silling, O. Weckner, E. Askari, F. Bobaru, Crack nucleation in a peridynamic solid, Int. J. Fract. 162 (2010) 219-227.

[6] F. Bobaru, M. Duangpanya, A peridynamic formulation for transient heat conduction in bodies with evolving discontinuities, Journal of Computational Physics 231 (2012) 2764-2785.

[7] F. Bobaru, M. Duangpanya, The peridynamic formulation for transient heat conduction, Int. J. Heat Mass Transfer 53 (2010) 4047-4059.

[8] F. Andreu-Vaillo, J. M. Mazón, J. D. Rossi, J. J. Toledo-Melero, Nonlocal Diffusion Problems, Vol. 165 of Mathematical Surveys and Monographs, American Mathematical Society, 2010.

[9] Q. Du, Z. Huang., R. B. Lehoucq, Nonlocal convection-diffusion volume-constrained problems and jump processes, Disc. Cont. Dyn. Sys. B 19 (2014) 373-389.

320 [10] P. Seleson, M. Gunzburger, M. L. Parks, Interface problems in nonlocal diffusion and sharp transitions between local and nonlocal domains, Comput. Methods Appl. Mech. Engrg. 266 (2013) $185-204$.

[11] B. Kilic, A. Agwai, E. Madenci, Peridynamic theory for progressive damage prediction in centercracked composite laminates, Composite Structures 90 (2009) 141-151.

[12] J. Xu, A. Askari, O. Weckner, S. Silling, Peridynamic analysis of impact damage in composite laminates, J. Aerosp. Eng., SPECIAL ISSUE: Impact Mechanics of Composite Materials for Aerospace Application, 21 (2008) 187-194.

[13] E. Oterkus, E. Madenci, O. Weckner, S. Silling, P. Bogert, A. Tessler, Combined finite element and peridynamic analyses for predicting failure in a stiffened composite curved panel with a central slot, Composite Structures 94 (2012) 839-850.

[14] E. Oterkus, E. Madenci, Peridynamics for failure prediction in composites, in: 53rd AIAA/ASME/ASCE/AHS/ASC Structures, Structural Dynamics and Materials Conference, Honolulu, Hawaii, 2012.

[15] W. Hu, Y. D. Ha, F. Bobaru, Peridynamic model for dynamic fracture in unidirectional fiberreinforced composites, Comput. Methods Appl. Mech. Engrg. 217-220 (2012) 247-261.

[16] B. Kilic, E. Madenci, Prediction of crack paths in a quenched glass plate by using peridynamic theory, Int. J. Fract. 156 (2009) 165-177.

[17] Y. D. Ha, F. Bobaru, Characteristics of dynamic brittle fracture captured with peridynamics, Engineering Fracture Mechanics 78 (2011) 1156-1168.

340 [18] D. J. Littlewood, A nonlocal approach to modeling crack nucleation in AA 7075-T651, in: Proceedings of the ASME 2011 International Mechanical Engineering Congress and Exposition, Denver, Colorado, American Society of Mechanical Engineers, 2011, pp. 567-576, Paper No. IMECE201164236.

[19] P. Seleson, M. L. Parks, On the role of the influence function in the peridynamic theory, International Journal for Multiscale Computational Engineering 9 (2011) 689-706.

[20] S. A. Silling, E. Askari, A meshfree method based on the peridynamic model of solid mechanics, Comp. Struct. 83 (2005) 1526-1535.

[21] M. R. Tupek, J. J. Rimoli, R. Radovitzky, An approach for incorporating classical continuum damage models in state-based peridynamics, Comput. Methods Appl. Mech. Engrg. 263 (2013) $20-26$. 
[22] F. Bobaru, Y. D. Ha, W. Hu, Damage progression from impact in layered glass modeled with peridynamics, Open Engineering 2 (2012) 551-561.

[23] E. Askari, F. Bobaru, R. B. Lehoucq, M. L. Parks, S. A. Silling, O. Weckner, Peridynamics for multiscale materials modeling, Journal of Physics: Conference Series 125 (1) (2008) 012078.

[24] M. Ghajari, L. Iannucci, P. Curtis, A peridynamic material model for the analysis of dynamic crack propagation in orthotropic media, Computer Methods in Applied Mechanics and Engineering 276 (2014) 431-452.

[25] D. J. Littlewood, K. Mish, K. Pierson, Peridynamic simulation of damage evolution for structural health monitoring, in: Proceedings of the ASME 2012 International Mechanical Engineering Congress and Exposition, Houston, Texas, American Society of Mechanical Engineers, 2012, pp. 1-8, Paper No. IMECE2012-86400.

[26] W. Gerstle, N. Sau, S. Silling, Peridynamic modeling of concrete structures, Nuclear Engineering and Design 237 (2007) 1250-1258.

[27] S. A. Silling, R. B. Lehoucq, Convergence of peridynamics to classical elasticity theory, J. Elasticity 93 (2008) 13-37.

[28] E. Emmrich, O. Weckner, On the well-posedness of the linear peridynamic model and its convergence towards the Navier equation of linear elasticity, Commun. Math. Sci. 5 (2007) 851-864.

[29] T. Mengesha, Q. Du, Nonlocal constrained value problems for a linear peridynamic Navier equation, Journal of Elasticity 116 (2014) 27-51.

30] P. Seleson, D. J. Littlewood, Convergence studies in meshfree peridynamic simulations, Computers \& Mathematics with Applications 71 (2016) 2432-2448.

[31] X. Tian, Q. Du, Analysis and comparison of different approximations to nonlocal diffusion and linear peridynamic equations, SIAM J. Numer. Anal. 51 (2013) 3458-3482.

[32] X. Tian, Q. Du, Asymptotically compatible schemes and applications to robust discretization of nonlocal models, SIAM J. Numer. Anal. 52 (4) (2014) 1641-1665.

[33] Q. Du, X. Tian, Asymptotically compatible schemes for peridynamics based on numerical quadratures, in: Proceedings of the ASME 2014 International Mechanical Engineering Congress and Exposition, Montreal, Quebec, Canada, American Society of Mechanical Engineers, 2014, p. V001T01A058, Paper No. IMECE2014-39620.

380 [34] M. A. Bessa, J. T. Foster, T. Belytschko, W. K. Liu, A meshfree unification: reproducing kernel peridynamics, Computational Mechanics 53 (2014) 1251-1264.

[35] F. Bobaru, M. Yang, L. F. Alves, S. A. Silling, E. Askari, J. Xu, Convergence, adaptive refinement, and scaling in 1D peridynamics, Int. J. Numer. Meth. Engng. 77 (2009) 852-877.

[36] J. A. Mitchell, S. A. Silling, D. J. Littlewood, A position-aware linear solid constitutive model for peridynamics, Journal of Mechanics of Materials and Structures 10 (5) (2015) 539-557.

[37] S. A. Silling, M. Epton, O. Weckner, J. Xu, E. Askari, Peridynamic states and constitutive modeling, J. Elasticity 88 (2007) 151-184.

[38] S. A. Silling, R. B. Lehoucq, Peridynamic theory of solid mechanics, Advances in Applied Mechanics 44 (2010) 73-168.

39] E. Madenci, E. Oterkus, Peridynamic Theory and Its Applications, Springer-Verlag, New York, 2014.

[40] M. L. Parks, P. Seleson, S. J. Plimpton, S. A. Silling, R. B. Lehoucq, Peridynamics with LAMMPS: A user guide v0.3 beta, SAND Report 2011-8523, Sandia National Laboratories, Albuquerque, NM and Livermore, CA (2011).

41] F. Bobaru, Y. D. Ha, Adaptive refinement and multiscale modeling in 2D peridynamics, International Journal for Multiscale Computational Engineering 9 (2011) 635-660.

[42] P. Seleson, Improved one-point quadrature algorithms for two-dimensional peridynamic models based on analytical calculations, Comput. Methods Appl. Mech. Engrg. 282 (2014) 184-217.

[43] Z. Chen, F. Bobaru, Selecting the kernel in a peridynamic formulation: A study for transient heat diffusion, Computer Physics Communications 197 (2015) 51-60.

[44] P. Seleson, Y.-D. Ha, S. Beneddine, Concurrent coupling of bond-based peridynamics and the Navier equation of classical elasticity by blending, Inter. J. Multiscale Comp. Engrg. 13 (2015) 91-113.

[45] G. C. Ganzenmüller, S. Hiermaier, M. May, Improvements to the prototype micro-brittle model of peridynamics, in: M. Griebel, M. A. Schweitzer (Eds.), Lecture Notes in Computational Science and Engineering, Springer International Publishing, Switzerland, 2015, Ch. 9, pp. 163-183.

[46] S. A. Silling, Linearized theory of peridynamic states, J. Elast. 99 (2010) 85-111.

[47] I. Jasiuk, J. Chen, M. Thorpe, Elastic moduli of two dimensional materials with polygonal and elliptical holes, Appl. Mech. Rev. 47 (1) (1994) S18-S28.

[48] J. E. Prussing, The principal minor test for semidefinite matrices, Journal of Guidance, Control, 
and Dynamics 9 (1) (1986) 121-122.

[49] M. L. Parks, R. B. Lehoucq, S. J. Plimpton, S. A. Silling, Implementing peridynamics within a molecular dynamics code, Comp. Phys. Comm. 179 (2008) 777-783.

[50] P. D. Seleson, Peridynamic multiscale models for the mechanics of materials: Constitutive relations, upscaling from atomistic systems, and interface problems, Ph.D. thesis, Florida State University, electronic Theses, Treatises and Dissertations. Paper 273. (2010).

51] S. F. Henke, S. Shanbhag, Mesh sensitivity in peridynamic simulations, Computer Physics Communications 185 (2014) 181-193.

[52] F. Bobaru, G. Zhang, Why do cracks branch? A peridynamic investigation of dynamic brittle fracture, Int. J. Fract. 196 (2015) 59-98.

420 [53] D. Dipasquale, G. Sarego, M. Zaccariotto, U. Galvanetto, Dependence of crack paths on the orientation of regular 2D peridynamic grids, Engineering Fracture Mechanics 160 (2016) 248-263. 